\title{
EFECTO DE SITIO EN TUXTLA GUTIÉRREZ, CHIAPAS, UN DETERMINANTE EN LOS DAÑOS HISTÓRICOS EN EDIFICACIONES
}

\author{
Raúl González Herrera (1), Juan Carlos Mora Chaparro ${ }^{(2)}$, Jorge Aguirre González ${ }^{(3)}$, \\ Jorge A. Aguilar Carboney ${ }^{(4)}$, Jorge A. Paz Tenorio ${ }^{(1)}$ y Roberto Moreno Ceballo ${ }^{(1)}$
}

\begin{abstract}
RESUMEN
El 7 de septiembre del año 2017 ocurrió el último sismo que generó daños en la ciudad de Tuxtla Gutiérrez, Chiapas. Al analizarlo en conjunto con los sismos históricos más importantes en la región (7 de abril de 2011, 21 de octubre de 1995 y 23 de septiembre de 1902), y localizando los daños mediante un Sistema de Información Geográfica, se observó que -no obstante que los sistemas estructurales asociados a las construcciones dañadas están presentes a lo largo de la mancha urbanalos daños se concentraron en zonas específicas de la ciudad. La presente investigación tiene como objetivo explicar las causas de los daños por sismo, encontrándose que la causa más determinante de éstos es el efecto de sitio presente en los suelos del valle de Tuxtla, los cuales presentan amplificaciones hasta tres veces la aceleración del sismo en roca determinada mediante los registros de los sismos históricos.
\end{abstract}

Palabras clave: efecto de sitio; daños por sismo; correlación de daños; amplificación en suelos blandos; peligro sísmico

\section{SITE EFFECT IN TUXTLA GUTIÉRREZ, CHIAPAS, A DETERMINANT IN THE HISTORICAL DAMAGES IN BUILDINGS}

\begin{abstract}
On September 7th, 2017, occurred the last earthquake that caused severe damages in the city of Tuxtla Gutiérrez, Chiapas. The evaluation of structural damages together with those observed on the most important earthquakes in the region (April 7th, 2011, October 21st, 1995 and September 23rd, 1902) and locating the damage by means of a Geographical Information System, it was observed that despite those structural systems associated with damaged buildings were similar on all the urban area, the
\end{abstract}

Artículo recibido el 17 de julio de 2018 y aprobado para su publicación el 27 de junio de 2020. Se aceptarán comentarios y/o discusiones hasta cinco meses después de su publicación.

(1) Universidad de Ciencias y Artes de Chiapas, Ciudad Universitaria, Libramiento Norte Poniente \# 1150, Tuxtla Gutiérrez, Chiapas, $\underline{\text { ingeraul@yahoo.com, raul.gonzalez@,unicach.mx }}$

(2) Instituto de Geofísica, Universidad Nacional Autónoma de México, Ciudad Universitaria, Coyoacán, C.P. 04510, Ciudad de México, jcmora@,igeofisica.unam.mx

(3) Instituto de Ingeniería, Universidad Nacional Autónoma de México, Ciudad Universitaria, Coyoacán, C.P. 04510, Ciudad de México,joagg@pumas.iingen.unam.mx

(4) Universidad Autónoma de Chiapas (UNACH), Facultad de Ingeniería, Campus I, Boulevard Belisario Domínguez, Tuxtla Gutiérrez, Chiapas, jcarboney@gmail.com

DOI: $10.18867 /$ ris. 102.511 
damage was concentrated in specific areas of the city. The investigation tried to explain the causes of those damages, finding that the most determining reason for earthquake damage is the site effect present in the soils of the Tuxtla Valley, which have amplifications up to three times the acceleration of the earthquake in rock determined by records of historical earthquakes.

Keywords: site effect; earthquake damage; damage correlation; amplification in soft soils; seismic hazard

\section{INTRODUCCIÓN}

El día 7 de septiembre de 2017, el Servicio Sismológico Nacional (SSN) reportó un sismo con magnitud Mw 8.2, localizado en el Golfo de Tehuantepec a $133 \mathrm{~km}$ al suroeste de Pijijiapan, Chiapas. El sismo, ocurrido a las 23:49:17 horas (04:49 tiempo universal coordinado por sus siglas en inglés UTM), fue sentido en el sur y centro de México, así como en países de Centroamérica. Las coordenadas del epicentro fueron $14.761^{\circ}$ latitud y $-94.103^{\circ}$ longitud y profundidad de $45.9 \mathrm{~km}$ (SSN, 2017; Okuwaki y Yagi, 2017; Ye et al., 2017), y el mecanismo focal del sismo mostró una falla normal (rumbo $=311^{\circ}$, echado $=84.4^{\circ}$, desplazamiento $=-94.7^{\circ}$ ), la cual, es característica de un sismo intraplaca. En esta región, la placa de Cocos se subduce por debajo de la placa de Norteamérica y Caribe (SSN, 2017).

De acuerdo con lo reportado en el boletín de la Sociedad Mexicana de Ingeniería Sísmica (SMIS, 2017); así como en redes nacionales (SSN, 2017) y locales en Tuxtla Gutiérrez, Chiapas (estación de la Red Interuniversitaria de Instrumentación Sísmica (RIIS) y del sistema de alertamiento sísmico municipal), se obtuvieron aceleraciones pico en la zona cercana al epicentro, tales como de $488.7 \mathrm{~cm} / \mathrm{s}^{2}$ en Santiago Niltepec, Oaxaca (figura 1). Estas aceleraciones son un indicador de la magnitud de los daños en la región Istmo de Tehuantepec, Oaxaca, región que concentró la mayor parte de la energía del evento y de las subsecuentes réplicas (SSN, 2017). Los datos para el estado de Chiapas se muestran en la tabla 1 y se constriñen a las dos principales ciudades del estado. En la figura 2 se representa la ubicación de las estaciones en Tuxtla Gutiérrez, Chiapas que registraron el sismo del 7 de septiembre de 2017.

Tabla 1. Relación de aceleración pico máxima para dos ciudades del estado de Chiapas (SSN, 2017; redes acelerográficas locales)

\begin{tabular}{clll}
\hline \multicolumn{1}{c}{ Municipio } & \multicolumn{1}{c}{ Ubicación } & \multicolumn{1}{c}{ Aceleración } & \multicolumn{1}{c}{ Red } \\
\hline \multirow{3}{*}{ Tuxtla Gutiérrez } & $\begin{array}{l}\text { Ciudad Universitaria, Universidad de Ciencias } \\
\text { y Artes de Chiapas (UNICACH) }\end{array}$ & $\begin{array}{l}70.3 \mathrm{~cm} / \mathrm{s}^{2} \\
204 \mathrm{~cm} / \mathrm{s}^{2} \text { (en entrepiso de } \\
\text { edificio de dos niveles) }\end{array}$ & $\begin{array}{l}\text { SSN } \\
\text { RIIS }\end{array}$ \\
\multirow{2}{*}{ Tuxtla Gutiérrez } & $\begin{array}{l}\text { Campus I. Universidad Autónoma de Chiapas } \\
\text { (UNACH) }\end{array}$ & $201 \mathrm{~cm} / \mathrm{s}^{2}$ & RIIS \\
& Edificio Torre Chiapas, Libramiento Norte & $162 \mathrm{~cm} / \mathrm{s}^{2}$ & $\begin{array}{l}\text { Red } \\
\text { municipal }\end{array}$ \\
Tuxtla Gutiérrez & Oriente & Red \\
& Edificio del Poder Judicial, Libramiento Norte & $158 \mathrm{~cm} / \mathrm{s}^{2}$ & municipal \\
Tapachula & Oriente & $110.7 \mathrm{~cm} / \mathrm{s}^{2}$ & SSN \\
& Área urbana & $108 \mathrm{~cm} / \mathrm{s}^{2}$ & IIUNAM \\
\hline
\end{tabular}




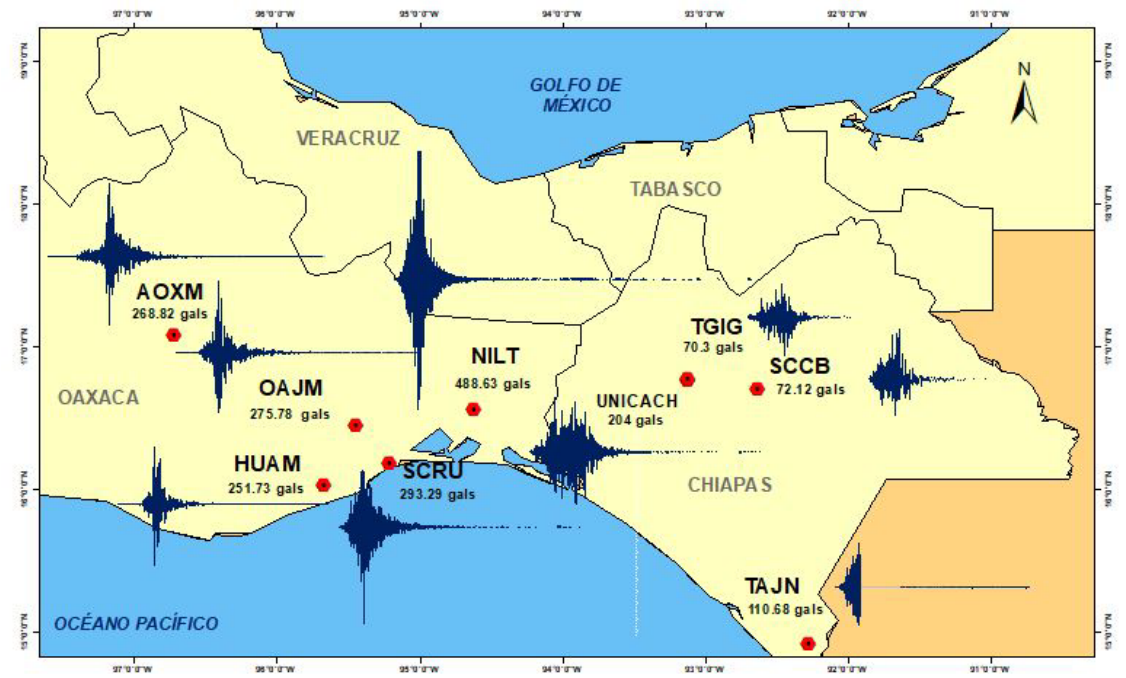

Figura 1. Mapa esquemático de aceleraciones registradas (información SSN, 2017 y datos de estación de RIIS en la UNICACH y de alertamiento sísmico municipal en Tuxtla Gutiérrez, Chiapas)

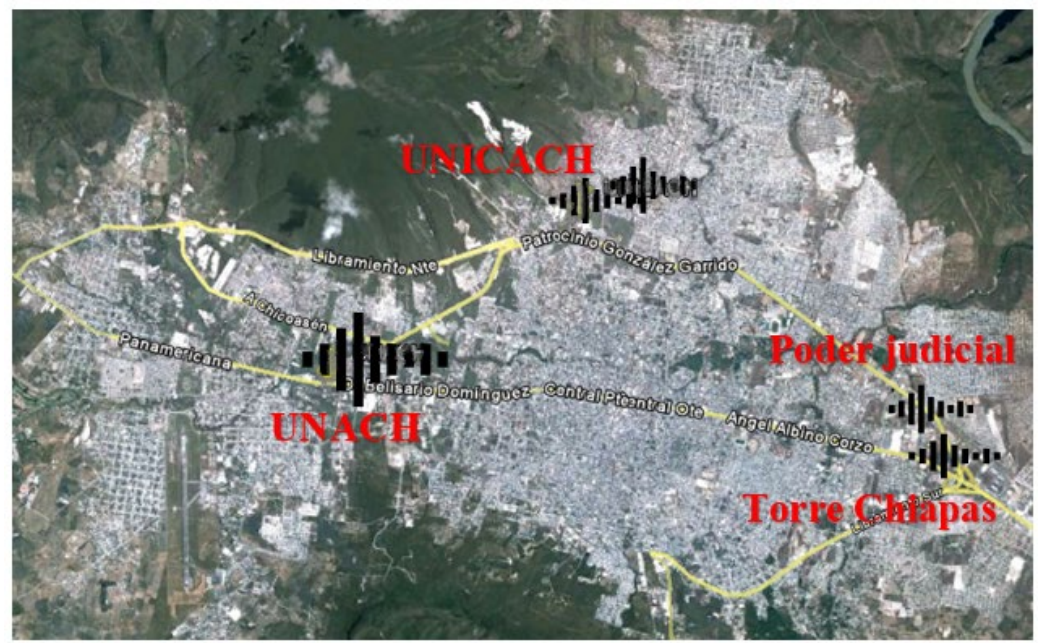

Figura 2. Ubicación de los acelerógrafos que registraron el sismo del 7 de septiembre de 2017 en Tuxtla Gutiérrez, Chiapas

Para desarrollar esta investigación se construyeron bases de datos de los daños por sismos, analizando documentos históricos, reportes de protección civil estatal y municipal, colegios de ingenieros civiles y arquitectos, entre otras. Las bases de datos que se construyeron para el sismo del 7 de septiembre de 2017 fueron desarrolladas a partir de información de entidades nacionales y locales, así como de trabajo de campo de los autores de este artículo. Los daños encontrados se correlacionaron contra parámetros físicos establecidos en la microzonificación sísmica de la ciudad (González-Herrera et al., 2012), misma que presenta mapas de isoperiodos, de amplificaciones con correlación de funciones de transferencia y estructura de velocidad en la zona.

Asimismo, y tras comparar los daños de los sismos más recientes que se tienen caracterizados en la ciudad de Tuxtla Gutiérrez ( 7 de abril de 2011 y del 21 de octubre de 1995; así como la probable ubicación de los daños durante el paleosismo del 23 de septiembre de 1902 (Figueroa, 1973)), con el empleo de Sistemas de Información Geográfica se observó que la ubicación de los daños coincide espacialmente con 
la de los producidos por el sismo reciente (7 de septiembre de 2017). Al analizar las consecuencias del sismo se observó que, no obstante que los sistemas estructurales asociados a las construcciones que fueron dañadas están presentes a lo largo de toda la mancha urbana, los daños se concentraron en zonas específicas de la ciudad, lo cual es un indicador importante del efecto de sitio.

\section{DAÑOS HISTÓRICOS E INTENSIDADES POR SISMO EN TUXTLA GUTIÉRREZ}

Un elemento clave a considerar para entender el peligro sísmico de una región son los daños históricos registrados. Éstos se pueden representar con un mapa de intensidades sísmicas, como el desarrollado por Figueroa (1986) utilizando 49 mapas de isosistas, que toman en cuenta los sismos más importantes ocurridos en nuestro país, entre el 7 de abril de 1845 y el 19 de septiembre de 1985. Los mapas de intensidades han sido empleados como elementos de entendimiento de la peligrosidad antes de la existencia de registros instrumentales y, a partir de ellos, relacionan las intensidades Modificadas de Mercalli (MMI) versus la aceleración pico del suelo (PGA), la velocidad pico del suelo (PGV) o el desplazamiento pico del suelo (PGD).

Gutiérrez et al. (1991) construyeron un sistema llamado Diagnóstico del Peligro Sísmico (DPS), el cual retoma los trabajos de isosistas de Figueroa (1986), para construir histogramas de intensidades de las distintas regiones del país. En este trabajo la ciudad de Tuxtla Gutiérrez alcanza intensidades sísmicas de Mercalli de X y Tapachula presenta intensidades de Mercalli VII. Las intensidades sísmicas, al estar directamente relacionadas con los daños observados, son un buen indicador del peligro sísmico, lo cual permite considerar que Tuxtla Gutiérrez presenta un nivel de peligro sísmico importante. La evolución del trabajo de Gutiérrez et al., se trasladó al Atlas Nacional de Riesgos desarrollado por CENAPRED (2015), el cual considera un periodo de estudio de las isosistas de sismos hasta el año 1999 y emplea 52 mapas de isosistas de sismos importantes (figura 3).

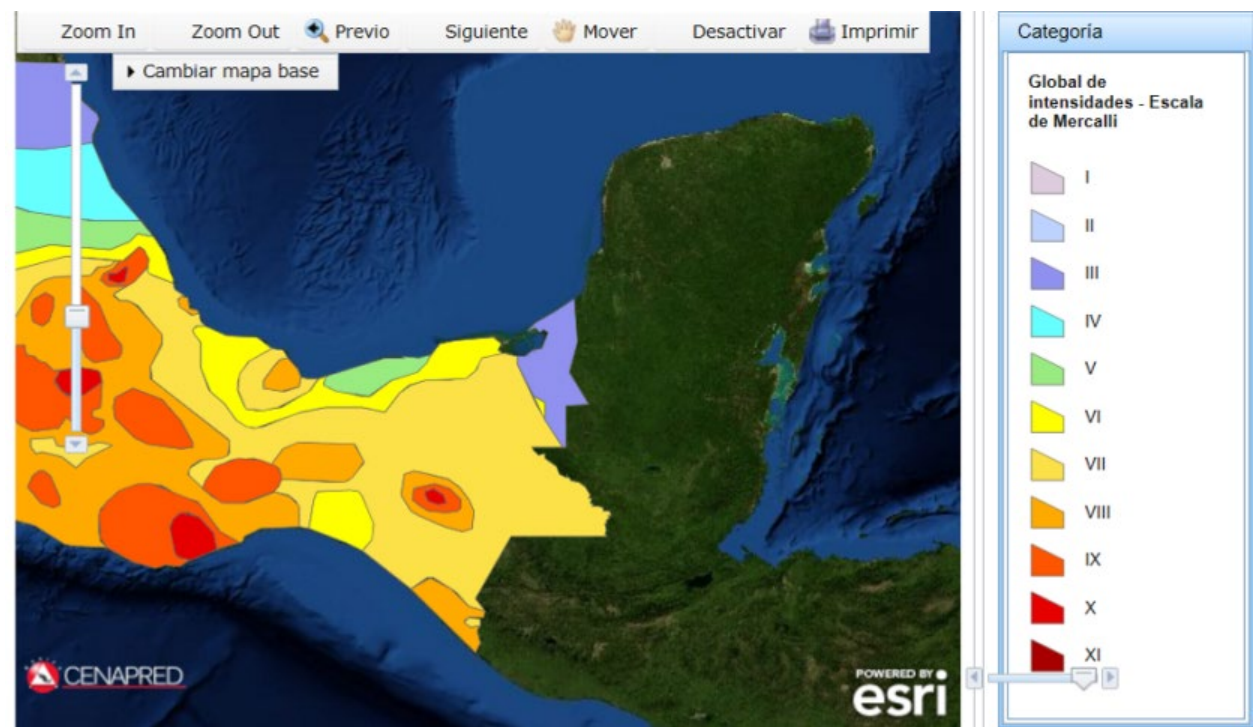

Figura 3. Mapa de intensidades sísmicas máximas basadas en la Escala Modificada de Mercalli para temblores de gran magnitud ocurridos entre 1845 y 1999 (CENAPRED, 2015)

De acuerdo con los trabajos de Castro (2001), García y Suárez (1996), González-Herrera et al. (2010, 2011a, 2011b, 2013) y González-Herrera (2014), en la ciudad de Tuxtla Gutiérrez se han presentado sismos que generaron daños moderados y hasta importantes. En el presente trabajo se consideran tres eventos 
históricos sísmicos particulares adicionales al sismo del 7 de septiembre de 2017, los cuales fueron seleccionados por la cantidad de daños generados y porque la información es suficiente para su localización, descripción y análisis, mismos que se describen a continuación:

- $\quad$ El 23 de septiembre de 1902 se presentó un sismo de subducción de la placa de Cocos por debajo de la placa Norteamericana, a $25 \mathrm{~km}$ de profundidad con magnitud de 7.7 e intensidades de Mercalli de X en la ciudad capital, alcanzando daños muy importantes y generalizados (Figueroa, 1973; García y Suárez, 1996). De acuerdo con los sistemas constructivos locales en esa época, los daños debieron presentarse prácticamente en todas las construcciones. Se considera que lo anterior es una de las principales evidencias de la carencia de construcciones históricas del siglo XIX o anteriores en Tuxtla Gutiérrez.

- El 21 de octubre de 1995 un fuerte sismo con epicentro cercano a Ocozocoautla, Chiapas, sacudió a Tuxtla Gutiérrez y la Región La Frailesca del estado, que fue la más afectada, cuya capital es Villaflores, lo que le dio el nombre al evento. El sismo presentó una profundidad de $165 \mathrm{~km}$ sin la generación de réplicas, lo anterior propició que los daños fueran menos importantes de los que hubo en la capital y regiones cercanas a ésta. Este evento fue de magnitud 7.2 e intensidad de Mercalli de VII en esta ciudad, ocasionando grietas en edificios y colapso de algunas viviendas, daños en gran parte de la infraestructura educativa, dos fallecimientos, la caída de bardas y pánico generalizado en la población (González-Herrera et al., 2011b) (figura 4).

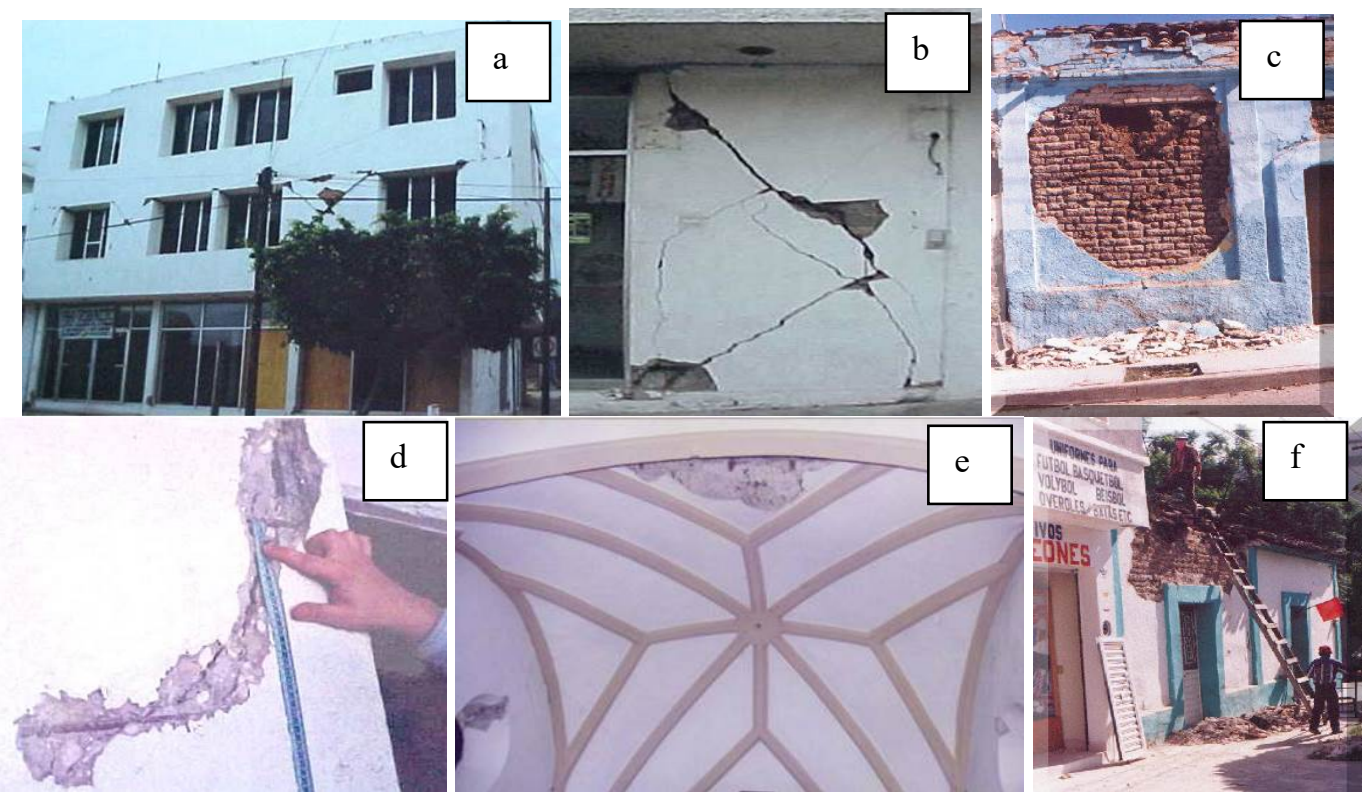

Figura 4. Fotografías de los daños debidos al sismo de Villaflores de octubre de 1995 en edificios de Tuxtla Gutiérrez (González-Herrera et al., 2011b). (a, b) Edificio público en el centro; (c) Estructura de adobe; (d) Rectoría de la UNACH; (e) Catedral de San Marcos; (f) Construcciones de adobe

- El tercer evento es significativo por los daños que produjo en la ciudad, no obstante, no ser una región cercana al epicentro. Este sismo se generó el 7 de abril de 2011, con una magnitud de 6.7 e intensidad de VI en regiones de suelo blando en Tuxtla Gutiérrez. Ocurrió a $83 \mathrm{~km}$ de las Choapas, Veracruz y propició en Tuxtla Gutiérrez un conjunto de daños en edificios públicos, viviendas, bardas, entre otras estructuras, los cuales no debieron haberse presentado por la distancia de la fuente, la profundidad $(167 \mathrm{~km})$ y la magnitud del evento (González-Herrera et al., 2012) (figura 5). Sin embargo, la 
presencia de éstos se debió al efecto de sitio en ciertas regiones de la ciudad donde se alcanzó una aceleración de $15 \%$ de la gravedad.
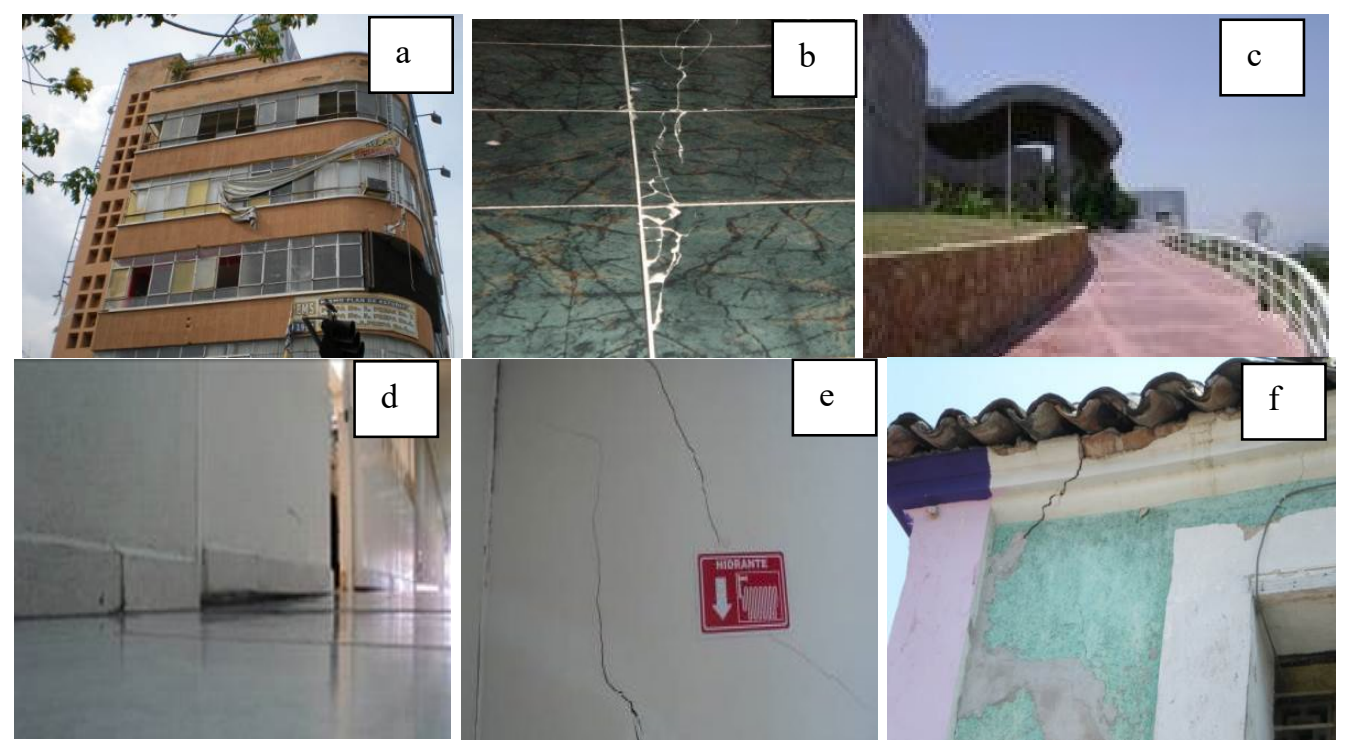

Figura 5. Fotografías de los daños causados por el sismo de Las Choapas de 2011 en Tuxtla Gutiérrez (González-Herrera et al., 2012). (a) Edificio Corzo; (b) Rectoría de la UNICACH; (c) COCYTECH; (d, e) Hospital de especialidades "Vida mejor"; (f) Vivienda en el centro de la ciudad

- $\quad$ El sismo del 7 de septiembre de 2017 produjo daños importantes en la ciudad. Los daños observados se atribuyen al efecto de sitio en la zona, a los materiales de construcción empleados, a la falta de mantenimiento, etc. Asimismo, la falta de recursos económicos y/o asesoría técnica es determinante, ya que la mayoría de los proyectos dañados son ejecutados mediante autoconstrucción, no solo de viviendas precarias, sino hasta residencias ejecutadas sin criterios ingenieriles y con materiales de baja calidad (Sánchez-Trujillo et al., 2019). En la figura 6 se observan algunos de los daños característicos observados durante el trabajo de campo.

\section{ANÁLISIS ESPACIAL DE LOS DAÑOS SÍSMICOS HISTÓRICOS PREVIOS AL SISMO DEL 7 DE SEPTIEMBRE DE 2017}

En la figura 7 se presenta un mapa donde se hace un análisis de los daños históricos de los sismos (23 de septiembre de 1902 -polígono verde, área que ocupaba la mancha urbana en 1892-; 21 de octubre de 1995 y 7 de abril de 2011), excluyendo el sismo del 7 de septiembre de 2017. Se observa que los daños se concentraron en la zona de suelos blandos conformados por arcillas expansivas, las cuales han sido estudiadas por Ordóñez (2008); Ordóñez-Ruiz et al. (2015) y se encuentran en el primer cuadro de la ciudad y zonas aledañas al río Sabinal y sus tributarios.

En dicho mapa (figura 7) se encontró que, en función del uso del inmueble, dentro de las estructuras dañadas, sobresalen las construcciones habitacionales y escolares de distintos niveles educativos. También es importante notar que los hospitales sufrieron daños menores, no obstante, se debió considerar esta experiencia para no repetirla en sismos futuros. Sin embargo, en el sismo del 7 de septiembre de 2017 nuevamente sufrieron daños hospitales públicos y privados (estos últimos, en ocasiones son construcciones adaptadas que no fueron diseñadas para tal fin) que habían sido reparados por daños de sismos previos, como hospitales de construcción reciente. 


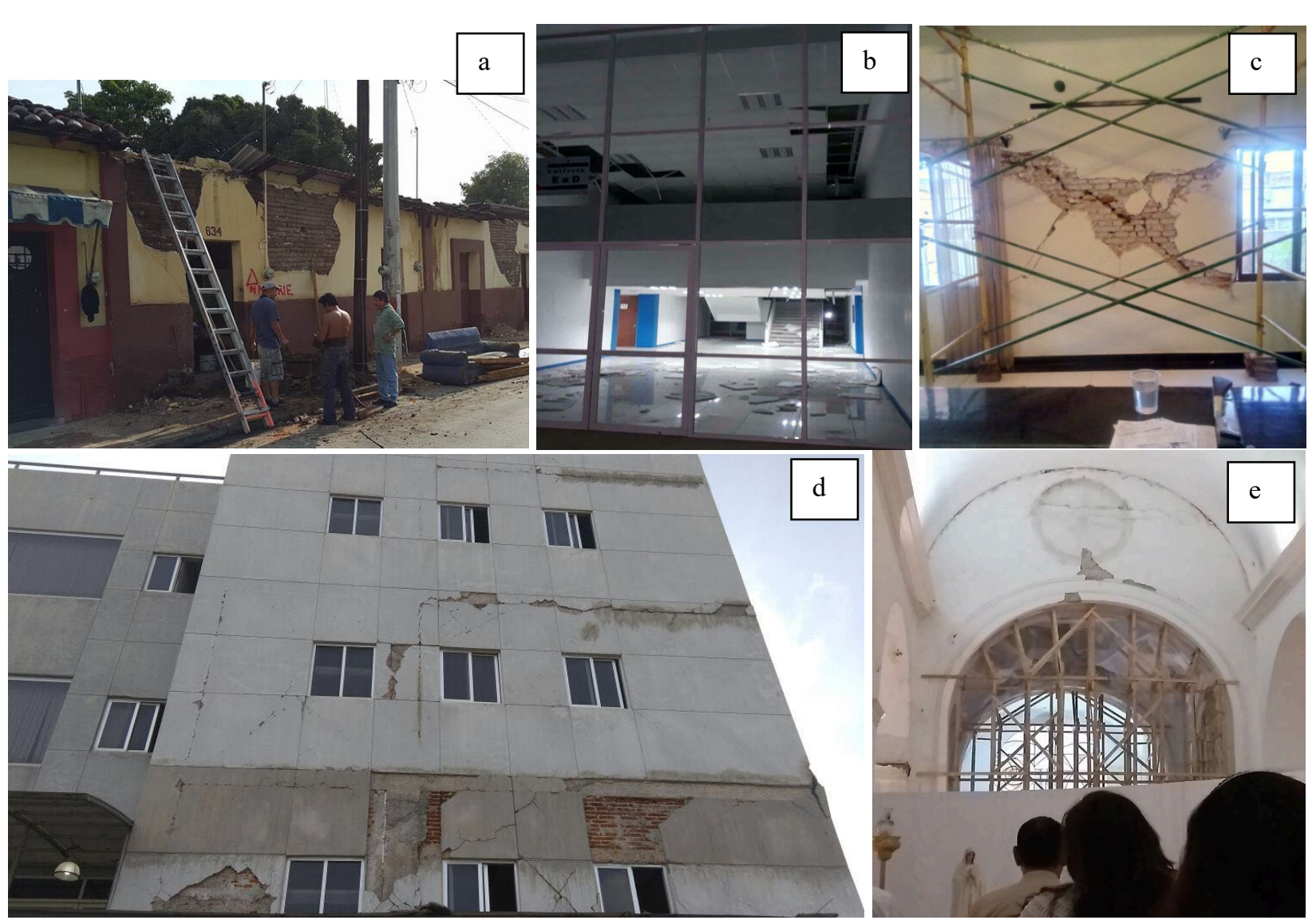

Figura 6. Fotografías de los daños causados por el sismo del Istmo de Tehuantepec de 2017 en edificación en Tuxtla Gutiérrez. (a) Viviendas de adobe; (b) Edificios públicos; (c) Residencia; (d) Edificio privado;

(e) Catedral de San Marcos

Considerando los daños históricos provocados por los sismos analizados en este trabajo, encontramos que otro factor importante en la concentración de daños en regiones específicas de la ciudad, es la edad de las construcciones. También se puede observar en la figura 7 que en el centro de la ciudad se concentraron daños y, de acuerdo con el estudio de González et al. (2010), en esta zona se registran construcciones con mayor antigüedad, que de acuerdo con el levantamiento físico estos daños corresponden a construcciones de entre 10 y 30 años. Las construcciones más jóvenes que sufrieron afectaciones corresponden a edificios ubicados en la cercanía de los tributarios del río Sabinal o construidos en las laderas, y obedecen -por lo general- a construcciones que no fueron diseñadas, construidas y/o supervisadas con asistencia ingenieril.

El periodo fundamental de las estructuras que sufrieron afectaciones corresponde a construcciones de uno a tres niveles que se asocian a periodos de entre 0.10 a $0.25 \mathrm{~s}$, de acuerdo con lo reportado en trabajos de Wilmer y Carrillo (2009), Aguilar (2006); y en el trabajo de campo realizado en González Herrera (2014). Las construcciones de tres o cuatro niveles que sufrieron daños se localizan en la cercanía del río Sabinal o sus tributarios. Algunas estructuras de más de cuatro niveles que fueron afectadas se localizan en el centro de la ciudad, donde los espesores de sedimentos son importantes y expansivos (González-Herrera et al., 2013; González-Herrera, 2014). 


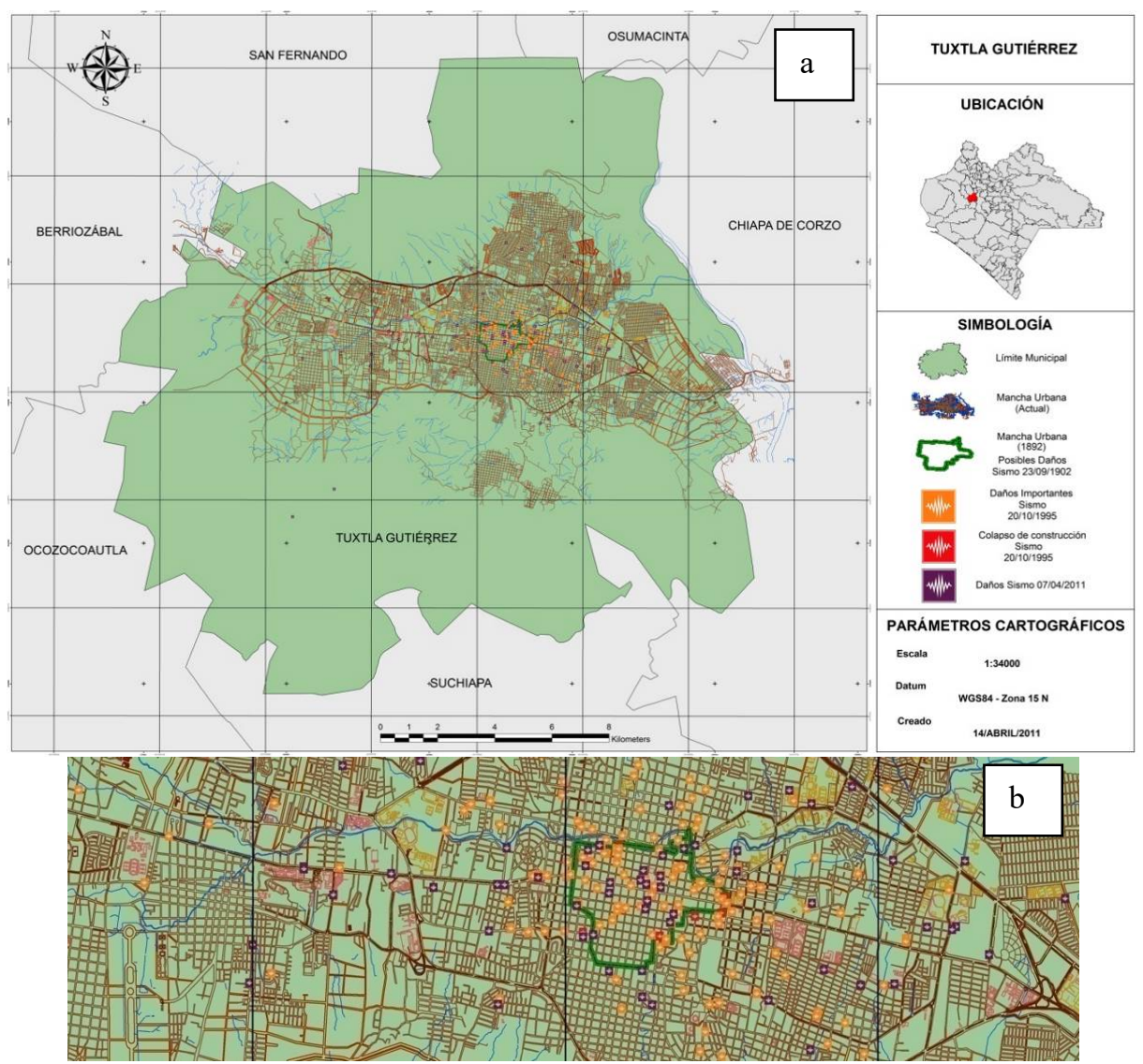

Figura 7. Daños históricos por sismo en Tuxtla Gutiérrez (González-Herrera et al., 2012). (a) Mancha urbana; (b) Centro de la ciudad. El polígono verde representa la mancha urbana de 1892 y posibles daños del sismo de 1902; puntos naranjas, daños del sismo de 1995 y puntos morados, daños del sismo del 7 de abril de 2011

Los sistemas estructurales que fueron afectados son variados (figura 8), entre los que predominan la mampostería sin refuerzo o con refuerzo insuficiente, así como construcciones de adobe y bajareque. Las estructuras de concreto y acero dañadas se asocian a errores en la construcción o en el diseño de acuerdo con el resultado de los peritajes realizados, posteriores a los sismos, por diversas instancias (Protección Civil, Colegio de Ingenieros Civiles y Colegio de Arquitectos). Las estructuras dañadas de mampostería se concentran en el centro de la ciudad, en San José Terán y en colonias residenciales del lado norte poniente y oriente, mientras que las estructuras dañadas de concreto y acero se distribuyen a lo largo del cauce del río Sabinal.

Las características del inventario de las construcciones en la zona de estudio, de acuerdo con diversos trabajos (González-Herrera et al., 2010; Arguello-Mendez, 2012; Sánchez-Trujillo et al., 2019), identifican que más de $90 \%$ de las edificaciones de la ciudad cuentan con alturas de hasta cuatro niveles, cuyos periodos fundamentales son similares a los del suelo, lo que permite generar fenómenos de coincidencia del periodo de las construcciones con el periodo fundamental del suelo. Esta coincidencia podría detonar en resonancia, de acuerdo con las características dinámicas de las construcciones y del suelo en que se desplantan. 


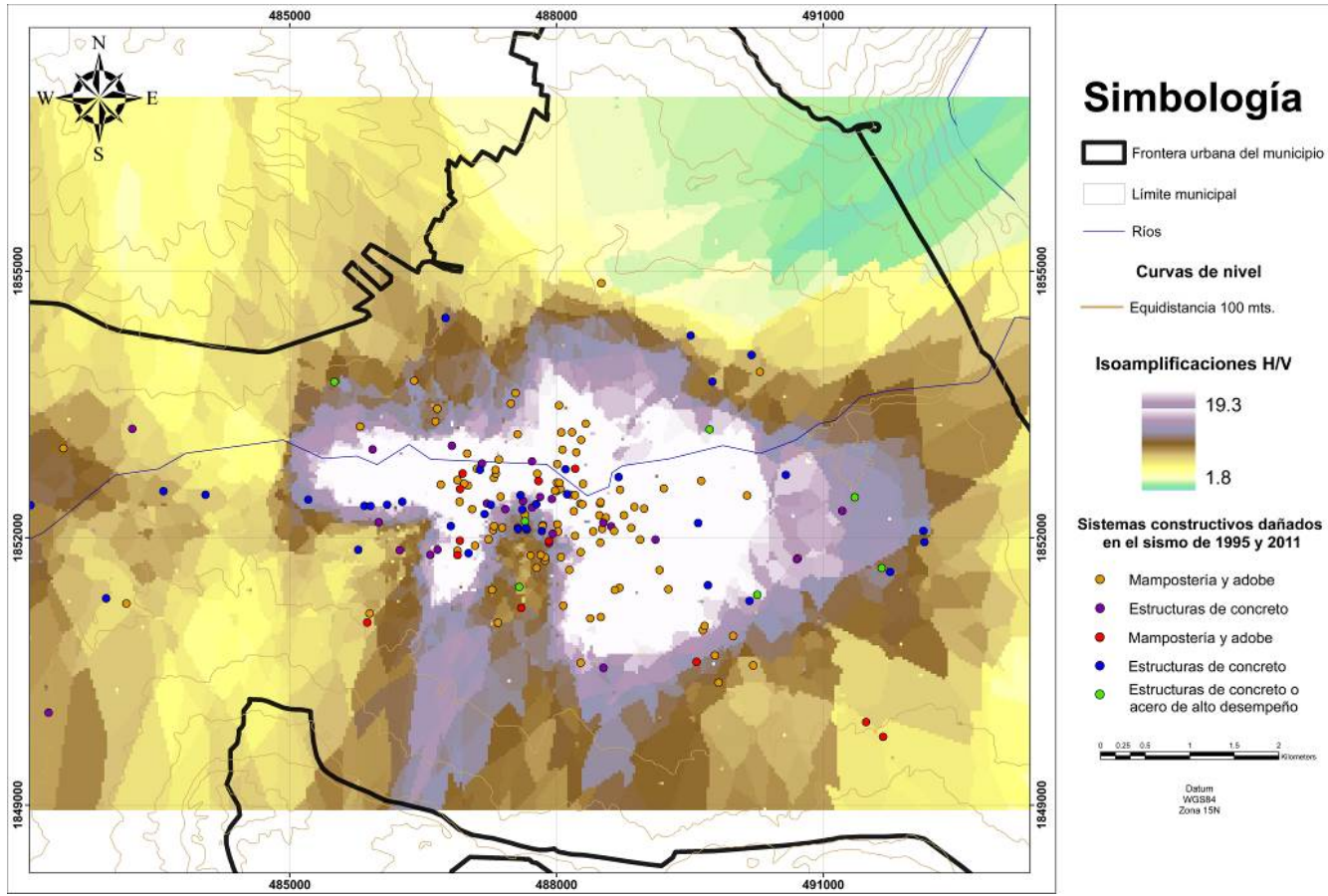

Figura 8. Localización de daños de los sismos históricos considerando los sistemas constructivos

En la figura 9 se muestra un análisis donde los puntos rojos corresponden a casos de daños donde el periodo del suelo y de la construcción son prácticamente los mismos, los puntos amarillos donde hay una diferencia desde $0.1 \mathrm{~s}$ hasta $0.15 \mathrm{~s}$ entre ambos periodos, $\mathrm{y}$ en punto verde donde hay diferencias superiores a $0.16 \mathrm{~s}$ entre ambos periodos. De acuerdo con la distribución espacial de las estructuras de mampostería en el centro de la ciudad, difícilmente fueron afectadas por el fenómeno de resonancia, no obstante, en la ladera sur existe una mayor probabilidad de que se haya presentado este fenómeno (puntos amarillos y rojos). Siguiendo con este análisis de distribución, las estructuras de concreto que pudieron ser afectadas por resonancia se concentran en el centro de la ciudad y aledañas al cauce del río Sabinal y se representan con punto rojo. Cabe mencionar que el área del mapa del valle de Tuxtla Gutiérrez y ladera sur, identificada con color blanco, es la zona donde se concentra la mayor parte de amplificación y de daños históricos por sismo.

\section{ANÁLISIS ESPACIAL DE LOS DAÑOS SÍSMICOS OCASIONADOS POR EL 7 DE SEPTIEMBRE DE 2017}

En párrafos anteriores se analizaron los daños sísmicos históricos en la ciudad de Tuxtla Gutiérrez, Chiapas, previos al sismo del 7 de septiembre de 2017. A continuación, se presenta un análisis de la concentración de daños históricos de los sismos analizados previamente, y se adicionan los del sismo del 7 de septiembre de 2017 (figura 10). En dicha figura se observa que los daños, tanto de los sismos previos como del sismo reciente, siguen un patrón recurrente y se concentran en el valle de Tuxtla Gutiérrez en tres regiones: El centro de la ciudad, San José Terán y zonas residenciales del norte oriente y poniente. Sin embargo, en el sismo del 7 de septiembre se desarrolló una tendencia de daños que no había sido identificada previamente, la cual se debe al crecimiento de la mancha urbana en esta región, que se ubica en los depósitos de talud de la ladera sur que presentan características geológicas y geotécnicas particulares (Paz et al., 2017; Ordóñez-Ruiz et al., 2015). 


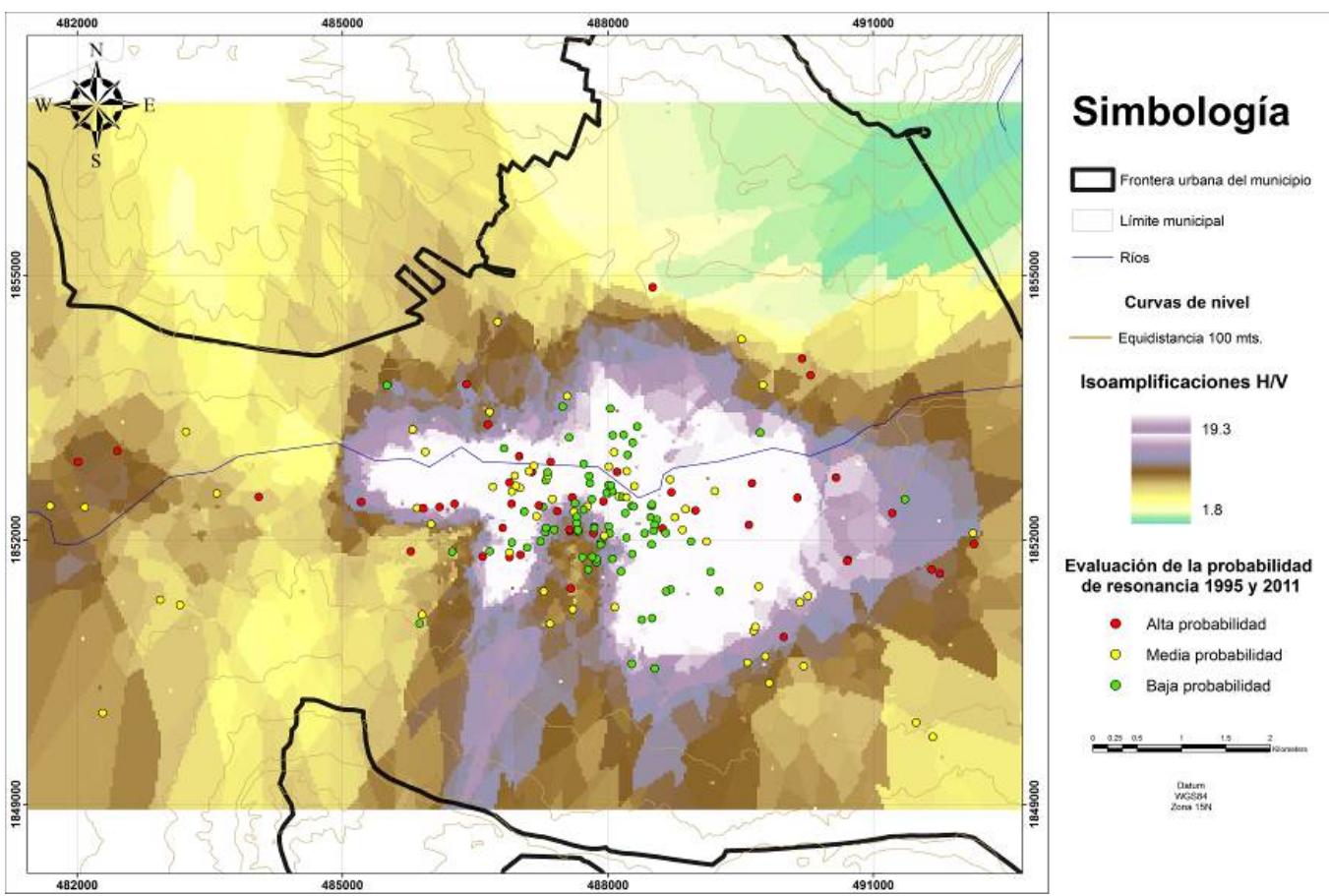

Figura 9. Localización de daños de los sismos históricos considerando la posibilidad de resonancia

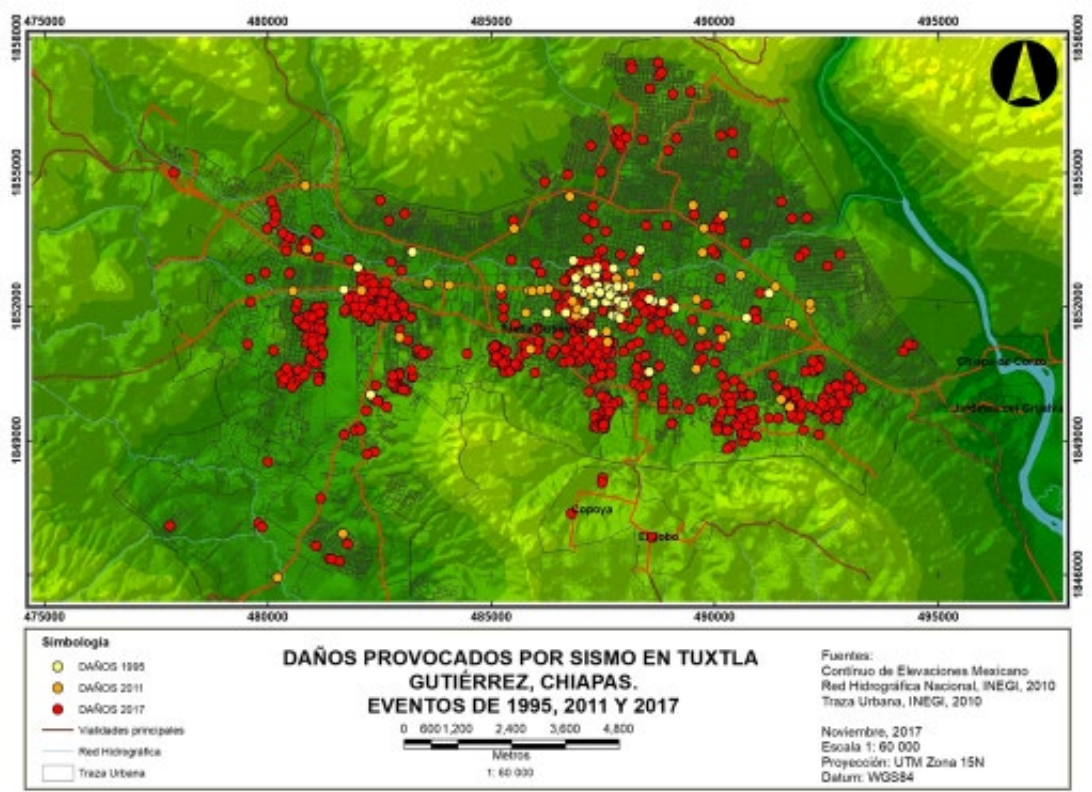

Figura 10. Localización de daños históricos de los sismos del 21 de octubre de 1995 (puntos amarillos), 7 de abril de 2011 (puntos naranjas) y 7 de septiembre de 2017 (puntos rojos)

En el estudio de riesgo sísmico desarrollado por Moreno et al. (2019) se propone que, en una ciudad con vulnerabilidad homogénea (en sus sistemas constructivos), el ubicar las zonas con efecto de sitio permite establecer con mayor certeza dónde podría haber daños por sismo. En caso de generar escenarios sísmicos con el propósito de gestión de riesgos, y asumiendo que se cumple la premisa anterior, estos modelos podrían ser calibrados a través de la base de datos de los daños históricos en la ciudad, en virtud de que donde se presentó daño, es debido a que hay efecto de sitio. González-Herrera (2014) propuso un escenario similar 
al del sismo de octubre de 1995 para las condiciones que predominaban en Tuxtla Gutiérrez en 2012 y obtiene el mapa mostrado en la figura 11, el cual alcanza una correlación del 67\% al considerar la concentración de daños que se presentaron en el sismo del 7 de septiembre de 2017.

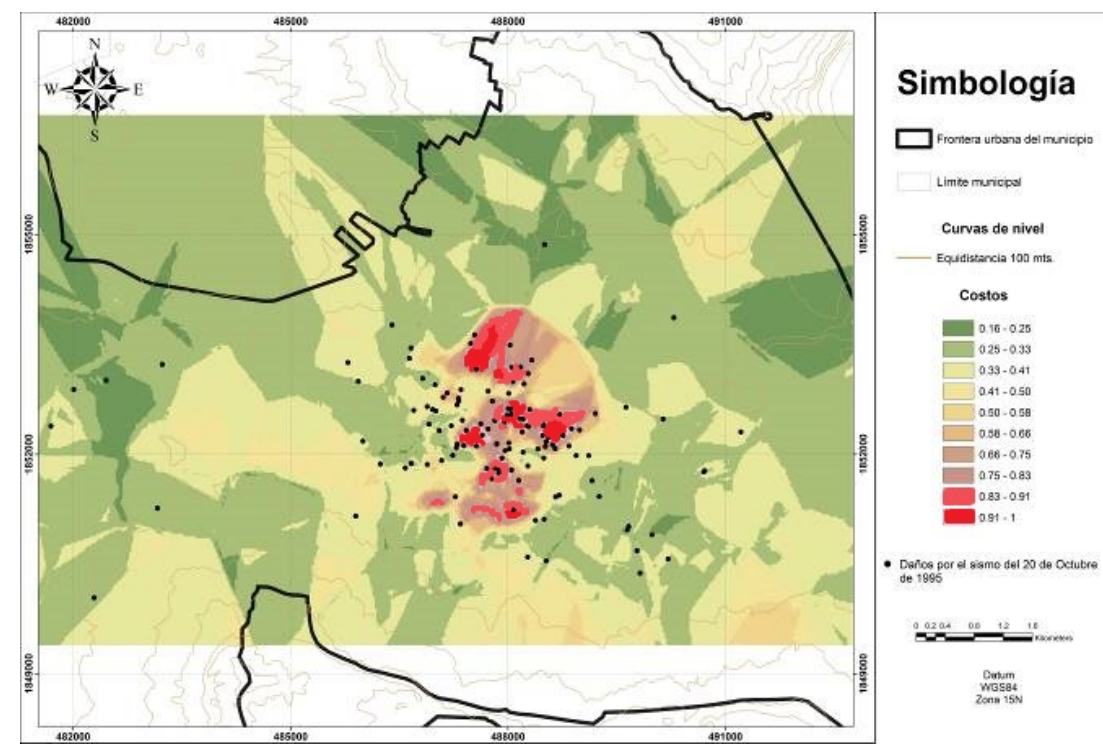

Figura 11. Escenario de riesgo sísmico para Tuxtla Gutiérrez que consideró aceleraciones pico similares a las presentadas $\left(200 \mathrm{~cm} / \mathrm{s}^{2}\right)$ en el sismo del 7 de septiembre de 2017

\section{EFECTO DE LOS EMBOVEDADOS EN LOS DAÑOS EN TUXTLA GUTIÉRREZ}

La ciudad de Tuxtla Gutiérrez se fundó en un valle fluvial sobre las márgenes del río Sabinal. Toda el agua de lluvia escurre por una serie de afluentes temporales y permanentes, hasta llegar al cauce principal del río Sabinal y de allí al río Grijalva. Desafortunadamente, los cauces naturales de estos escurrimientos fueron cubiertos en forma de embovedados o rellenados por el crecimiento de la mancha urbana (GonzálezHerrera, 2014). En la figura 12 se presenta un plano de localización de los principales embovedados con líneas de color rojo, que abarca las zonas de los barrios de San Marcos, El Calvario, San Pascualito, Los Milagros, Barrio Guadalupe, Fraccionamiento San Roque, Colonia Moctezuma y Colonia las Palmas.

Dichos embovedados presentan un deterioro importante en su estructura, debido a la falta de mantenimiento, edad y esfuerzos a los que se les somete durante su operación (figura 13). González-Herrera et al. (2013) realizaron un análisis de los principales embovedados, mediante una variación del método de Autocorrelación Espacial (SPAC) denominado Calicatas-SPAC. Mediante esta técnica se evaluó la ubicación de algunos de los embovedados y su relación con los daños presentados en los sismos históricos. La participación de los embovedados en los daños fue menor de lo que se esperaba y solo se ve influencia de éstos en los daños en las construcciones con las que colindan. 


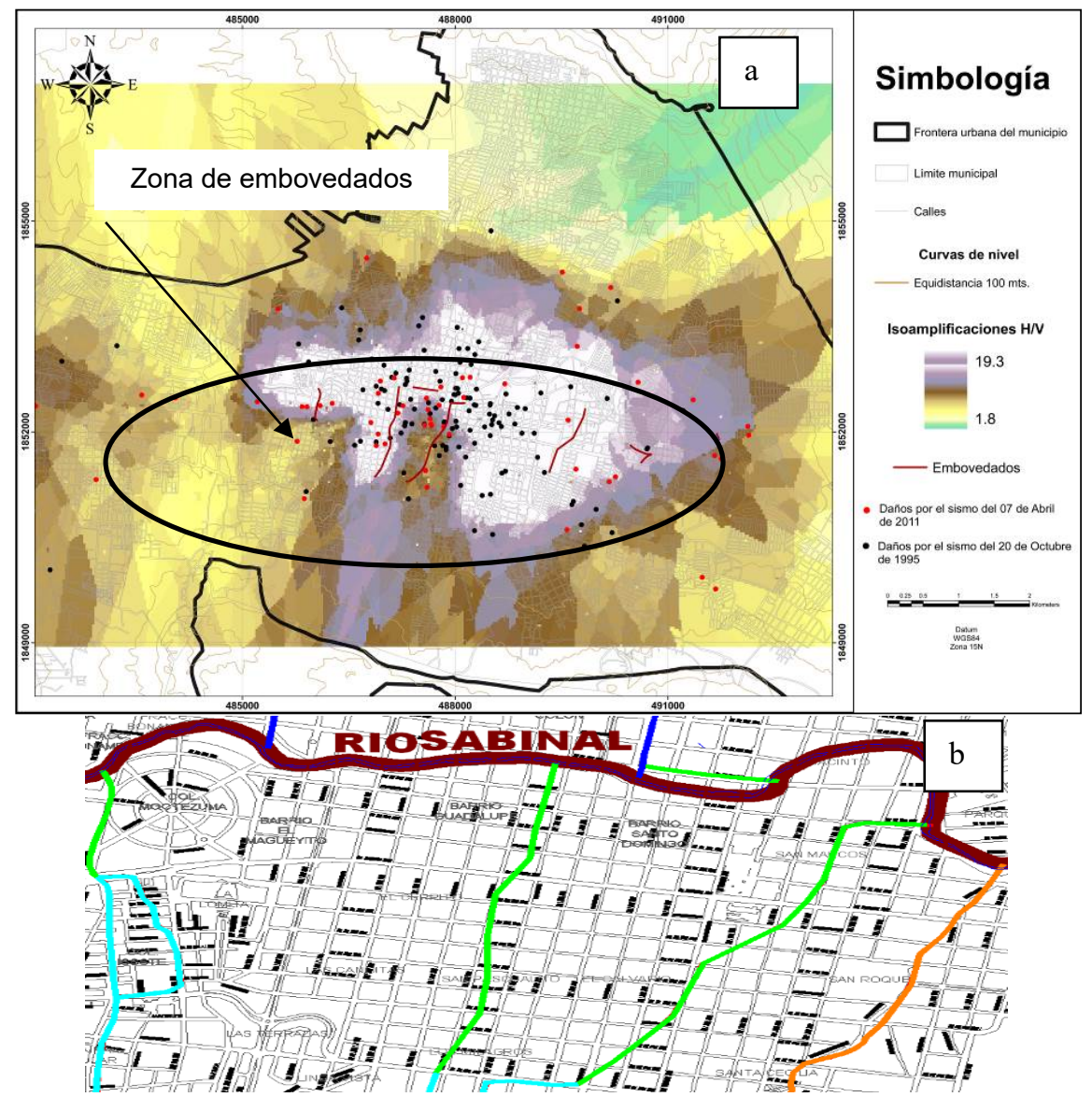

Figura 12. (a) Mapa de toda la ciudad; (b) Acercamiento del centro de Tuxtla Gutiérrez. Se muestra la ubicación de los embovedados siguiendo la trayectoria de las líneas color verde
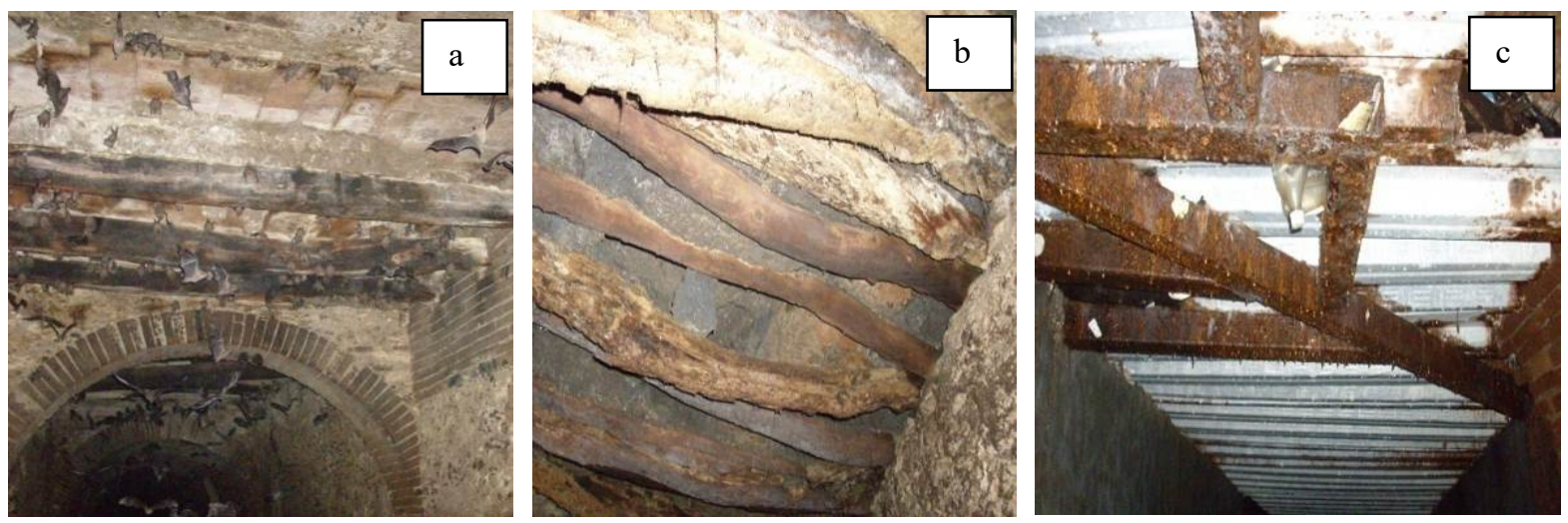

Figura 13. (a) Deterioro en estructura de embovedado de $4^{\mathrm{a}}$ Norte y $4^{\mathrm{a}}$ Oriente; (b) Corrosión en estructura de embovedado del barrio San Roque; (c) Deterioro y corrosión de la estructura de embovedado de 5a Poniente en Barrio Guadalupe. Fotografías de Protección Civil Estatal

\section{ANÁLISIS DEL EFECTO DE SITIO EN TUXTLA GUTIÉRREZ, CHIAPAS}

A mediados del siglo XX en México ya se conocía que el movimiento sísmico no se producía de igual forma en todas las zonas del terreno (Marsal y Mazari, 1959). En ese tiempo se había observado que en 
algunas áreas se generaban concentraciones de daños mayores que en otras. El origen de esta concentración se debe al fenómeno de amplificación y al periodo fundamental del movimiento del suelo, el cual aumenta tanto la aceleración, como la velocidad y el desplazamiento de la superficie en puntos concretos del terreno. Esta amplificación puede deberse a la naturaleza, composición y morfología de las capas superficiales del subsuelo. Al conjunto de estos fenómenos se le conoce como "efectos locales", "respuesta local" o "efectos del sitio” (Thomson, 1950; Haskell, 1953; Ewin et al., 1957; Haskell, 1960; Haskell, 1962; Aki y Richards, 1980).

Atakan et al. (1997) y Lermo y Chávez-García (1993), han demostrado que, durante la ocurrencia de un evento sísmico, la señal medida sobre un depósito de suelo presenta diferencias entre su duración, amplitud y contenido frecuencial respecto a una señal medida en el basamento rocoso, y esto se debe al cambio que sufren las ondas sísmicas al atravesar los distintos estratos de suelo. Estos cambios o amplificaciones de las ondas sísmicas están intrínsecamente relacionados al tipo del movimiento del sismo, topografía, geomorfología y a las propiedades mecánicas de los depósitos de suelos donde es medida la onda sísmica (Gallo et al., 2017).

En la actualidad es común estudiar los efectos de sitio en dos vertientes: La primera consiste en estimar las frecuencias a las cuales se espera una amplificación y la amplitud de ésta, y se analiza empleando los registros de sismos o registros de vibración ambiental; la otra vertiente consiste en determinar la geometría y las propiedades mecánicas del subsuelo, y emplea una simulación numérica para extrapolar valores y determinar los efectos de sitio (Chávez-García y Montalva, 2014).

Los estudios de microzonificación sísmica consisten en la identificación y caracterización de unidades de suelos y unidades litológicas subyacentes, para determinar la respuesta dinámica de los suelos frente a terremotos, esta respuesta constituye el efecto de sitio. Además de los suelos, en un estudio de microzonificación se incluyen los efectos inducidos por fallas, licuación y otros, y se valora su peligro. Los mapas resultantes, o mapas de microzonificación, se presentan en una base cartográfica útil para fines de edificación y planificación urbana (Tupak, 2009).

\section{Estudios de microzonificación sísmica en Tuxtla Gutiérrez}

La UAM Azcapotzalco (Alonso et al., 1995; Alonso et al., 1999) coordinó un equipo de especialistas que, en conjunto con académicos locales (UNACH), desarrollaron el primer mapa de microzonificación para Tuxtla Gutiérrez. La técnica empleada fue vibración ambiental, donde se estudiaron 75 puntos de la ciudad considerando espectros de Fourier; los trabajos incluyeron un mapa de isoperiodos que identificó un suelo homogéneo con tres isocurvas de $0.15,0.20$ y $0.25 \mathrm{~s}$. Con base en esto se propuso un espectro de diseño para suelo firme con amortiguamiento crítico del 5.0\% y con un coeficiente sísmico de 0.60 .

Los estudios de microzonificación para la ciudad de Tuxtla Gutiérrez, Chiapas continuaron en 2004, con trabajos desarrollados por la UNACH y la UNICACH (Narcía et al., 2006). Mediante estos estudios se complementó el mapa de microzonificación, considerando las nuevas colonias y refinando puntos del estudio previo (se pasó de 75 a 96 puntos de medición). El estudio encontró que el periodo natural de los suelos de Tuxtla Gutiérrez oscila desde 0.10 hasta 0.53 s, con amplitudes del orden de 13.

El estudio de Narcía et al. (2006) empleó la técnica de cocientes espectrales de Nakamura (1989), y retoma las mediciones de Alonso et al. (1995), algunas de las cuales no contaban con componente vertical por condiciones del equipo usado, para resolverlo se empleó una técnica basada en un modelo de Newmark y Rosenblueth (1976), la cual relaciona el periodo fundamental del suelo y la velocidad de las ondas de corte. Con esta solución se obtuvo la variación de los espesores de sedimentos que aportan al efecto de sitio, 
los cuales van desde $3.82 \mathrm{~m}$ hasta $19.95 \mathrm{~m}$. Se concluyó que el terreno debe clasificarse como firme para la mayoría de las laderas e intermedio para el valle.

Adicionalmente, se realizó una actualización de la microzonificación de Tuxtla Gutiérrez con trabajo de campo durante el año 2011, mediante la técnica de microtremores (vibración ambiental), empleando cocientes espectrales entre las componentes horizontales y la vertical (técnica de Nakamura, $H / V$, Nakamura, 1989). Lermo y Chávez-García (1994) compararon resultados de microtremores contra el cociente espectral estándar de registros de temblores y concluyeron que los microtremores, cuando son analizados con dicha técnica, determinan -con buena precisión- el periodo dominante $\left(T_{o}\right)$ en sedimentos sujetos a amplificación dinámica en un intervalo de frecuencias entre $0.5 \mathrm{~Hz}$ y $10 \mathrm{~Hz}$.

Para la adquisición de estos registros se utilizaron dos grabadoras Altus modelo Makalu con sensores modelo Episensor con rango de sensibilidad de $-1 / 4$ de $\mathrm{g}$ hasta $4 \mathrm{~g}$ y sensores de velocidad Lenartz de un segundo. Los registros se tomaron con 200 muestras cada segundo a lo largo de 30 minutos de muestreo por cada punto. En la distribución de estos registros se consideró la densidad de población, distancia entre 200 y $400 \mathrm{~m}$ entre los distintos puntos, buscando tener una interpolación adecuada, siendo el centro de la ciudad donde se densificaba la toma de muestras debido a los daños históricos por sismos (García y Suárez, 1996). En la figura 14 se muestran las curvas de isoperiodos para la ciudad. La amplificación relativa varía desde 2 hasta 25 veces, en una distribución dispersa en toda la ciudad.

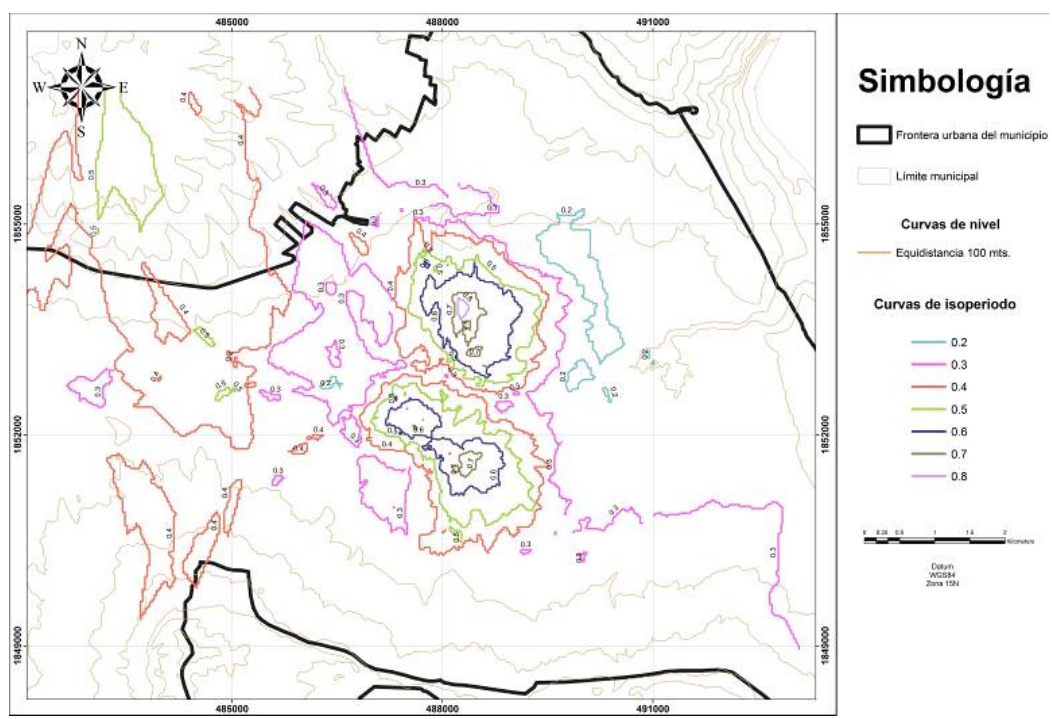

Figura 14. Mapa de isoperiodos de Tuxtla Gutiérrez (González-Herrera et al., 2012)

Dentro de los registros se seleccionaron ventanas de entre $30 \mathrm{~s}$ de duración, las cuales se utilizaron para calcular espectros de Fourier. Los resultados mostraron variación en sus frecuencias dominantes de 1 a $15 \mathrm{~Hz}$ ( 1 a $0.07 \mathrm{~s}$ de periodo) y una amplificación relativa máxima promedio de hasta 25 veces. Sin embargo, estas amplificaciones han de tomarse con reserva debido a que se han reportado que las reales pueden ser mayores (Bard, 1999). Los periodos de 0.51 hasta $1 \mathrm{~s}$, se presentaron principalmente en el centro y otras zonas de la ciudad cercanas al río Sabinal y sus tributarios. Lo anterior es ocasionado por un mayor espesor de sedimentos deformables, material constitutivo menos denso conformado básicamente por arcillas expansivas, entre otros factores. 


\section{Determinación de la estructura de velocidades mediante el método de Autocorrelación Espacial (SPAC)}

Los microtremores están conformados básicamente por ondas superficiales, y para su análisis se considera que son estacionarios en el espacio y tiempo. Aki (1957) propuso el método SPAC para obtener el modelo estructural de velocidades, para lo cual se requiere registrar microtremores simultáneamente en por lo menos dos estaciones que conforman un arreglo instrumental equidistante. Tras procesar la información se obtiene la curva de dispersión de ondas Rayleigh, que posteriormente se usa para determinar la estructura de velocidades (Aguirre et al., 2006).

Chávez-García et al. (2005) presentaron una extensión del método SPAC, donde las curvas de dispersión de velocidad de fase fueron obtenidas a partir de los registros de datos de un arreglo sísmico temporal con una geometría irregular. La diferencia básica con respecto al método propuesto por Aki (1957), fue el sustituir el promediado azimutal por el promediado temporal. Los resultados obtenidos en ese trabajo condujeron a proponer arreglos de estaciones que podían tener distintas geometrías, por ejemplo, una línea recta (Chávez-García et al., 2006), permitiendo liberar al método SPAC de las restricciones geométricas en la forma del arreglo usado (Aguirre et al., 2006; Flores, 2004).

En Tuxtla Gutiérrez, como parte de la actualización de la microzonificación, se realizaron un conjunto de arreglos SPAC, a partir de un trabajo de campo en 2011, considerando ocho diferentes arreglos. Adicionalmente, se incluyeron dos perfiles de velocidades obtenidos de estudios de efecto de sitio desarrollados en el Hospital Bicentenario, en la zona norte oriente (Aguilar et al., 2009), y cerca de las instalaciones de Home Depot, zona sur poniente de la ciudad (Aguilar et al., 2006), así como mediciones en donde se ubican las dos estaciones acelerométricas fijas en la ciudad.

El interés por conocer la estructura de velocidades de los puntos seleccionados para el estudio se debió a que en cinco de los puntos se observaron daños estructurales asociados a efecto de sitio, de acuerdo con estudios anteriores (Narcía et al., 2006; Piña, 2012). Los tres arreglos restantes (estacionamiento del estadio, Facultad de Ingeniería de la UNICACH y cercanías de la Torre Chiapas) consistieron en polígonos de hasta $400 \mathrm{~m}$ por lado, constituidos por triángulos equiláteros convencionales y correlaciones lineales. Dos sitios adicionales corresponden a la ubicación de las estaciones acelerométricas fijas en la ciudad (UNICACH, sede ladera norte y UNACH, poniente de la ciudad).

Respecto al equipo de adquisición de datos, los registros se recopilaron con dos consolas Kinemetrics, modelo Makalu de seis canales de 24 bits con sistema electrónico. A estos equipos se les conectó de manera externa un sensor triaxial marca EpiSensor modelo FBA ES-T, uno triaxial de velocidad marca Lennartz electronic LE-3Dlite MKII y una antena GPS.

Las gráficas mostradas en la figura 15, presentan la superposición de los perfiles de velocidades realizados y en ellas se observan, de manera global, los tipos de perfiles en que se puede clasificar el comportamiento sísmico de la ciudad y su correlación con las amplificaciones relativas reportadas (González-Herrera et al., 2013).

Para evaluar el peligro sísmico, considerando los efectos de sitio, se empleó el programa PRODISIS del Manual de Obras Civiles de CFE (2015) el cual permite obtener un espectro de diseño en roca para peligro uniforme. Adicionalmente, se puede introducir el perfil de velocidad $V s$ para considerar los efectos de sitio. Cabe mencionar que este manual de diseño sísmico es empleado en el estado de Chiapas al carecer éste de normas técnicas complementarias al reglamento de construcción. 

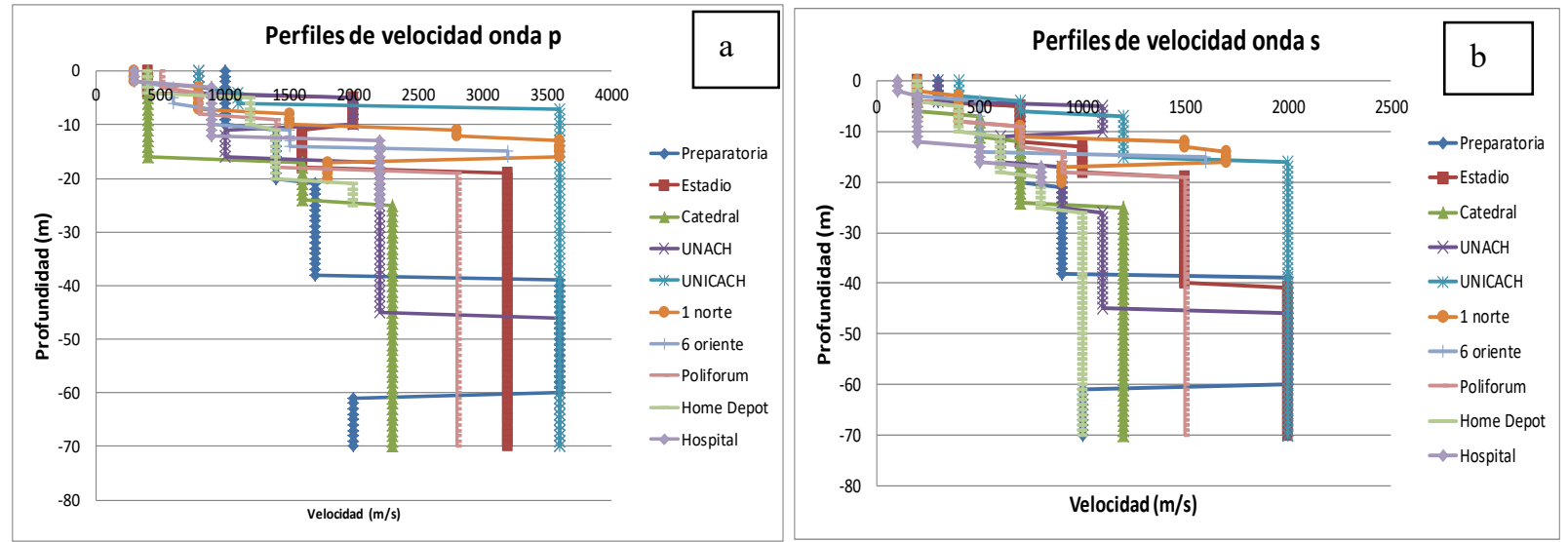

Figura 15. (a) Perfiles de velocidades Vp; (b) Perfiles de velocidades Vs, en Tuxtla Gutiérrez

Para conocer el efecto de sitio se introdujo en el software PRODISIS (CFE-2015) el perfil promedio de velocidades de la zona centro, obteniendo así un espectro de diseño que incluye el efecto de sitio. Al incluir el efecto de sitio, los espectros de diseño obtenidos con PRODISIS presentan amplificaciones del orden del $45 \%$. El basamento ingenieril, o suficientemente rígido para la cimentación de construcciones, se encuentra en los puntos que rebasan las velocidades de $720 \mathrm{~m} / \mathrm{s}$, los cuales se asocian con profundidades en un intervalo de 6 a $10 \mathrm{~m}$ en los sitios donde se realizaron los estudios (salvo en $6^{\mathrm{a}}$ oriente y catedral, en el centro; y Home Depot, en la ladera sur, que alcanzan hasta $30 \mathrm{~m}$ ). Realizando una correlación entre los distintos perfiles $V S$, considerando la profundidad en que esta velocidad alcanza el basamento ingenieril $(720 \mathrm{~m} / \mathrm{s})$ y normalizando este valor con respecto al punto más rígido entre las mediciones realizadas, se encontró que las instalaciones de la UNICACH (en el libramiento norte poniente) alcanzan dicho basamento a $6 \mathrm{~m}$ de profundidad. Con base en esto se desarrolló la tabla 2 y la figura 16, donde se correlaciona espacialmente esta normalización. González-Herrera et al. (2013) utilizan esta metodología para Tuxtla Gutiérrez, Chiapas empleando el Manual de Obras Civiles de CFE (2008) presentando mayor detalle del análisis de los datos obtenidos con PRODISIS.

Tabla 2. Estudios SPAC y su relación con las propiedades dinámicas del suelo en Tuxtla Gutiérrez

\begin{tabular}{|c|c|c|c|}
\hline Lugar & $\begin{array}{c}\text { Periodo } \\
\text { (s) }\end{array}$ & $\begin{array}{c}\text { Amplificación } \\
\text { relativa } \mathrm{H} / \mathrm{V}\end{array}$ & $\begin{array}{l}\text { Normalización } \\
\mathrm{H} / \mathrm{Vs}=720 \mathrm{~m} / \mathrm{s}\end{array}$ \\
\hline Ciudad Universitaria, UNICACH & 0.2 & 2 & 1.0 \\
\hline Preparatoria CETIS 138 & 0.8 & 11 & 3.3 \\
\hline Campo de futbol de facultad de Ingeniería de la UNACH & 0.5 & 4 & 2.8 \\
\hline Polifórum, Chiapas & 0.3 & 8 & 3.5 \\
\hline Calicatas-SPAC en $6^{\mathrm{a}}$ Oriente Norte entre 12 y 13 Norte* & 0.6 & 16 & 2.4 \\
\hline Calicatas-SPAC en $1^{\text {a }}$ Nte Poniente entre 12 y 13 Poniente & 0.6 & 14 & 2.1 \\
\hline Estacionamiento estadio de futbol Víctor Manuel Reyna & 0.7 & 10 & 2.9 \\
\hline Explanada de la Catedral de San Marcos & 0.6 & 8 & 2.4 \\
\hline Hospital de especialidades Bicentenario & 0.4 & 4 & 2.8 \\
\hline Home Depot Libramiento Sur Poniente & 0.3 & 7 & 3.4 \\
\hline
\end{tabular}

* En este punto se desarrollaron dos tipos de arreglos SPAC: Calicatas-SPAC y correlación lineal.

En la figura 16 se observa que existe correlación entre los daños y las amplificaciones obtenidas para los casos donde los daños se ubican en la zona localizada entre los intervalos 2 y 3 de la normalización, mismos que corresponden a profundidades entre 12 y $18 \mathrm{~m}$. Estas profundidades son características de estratos saturados de arcillas expansivas (Ordóñez, 2008). 


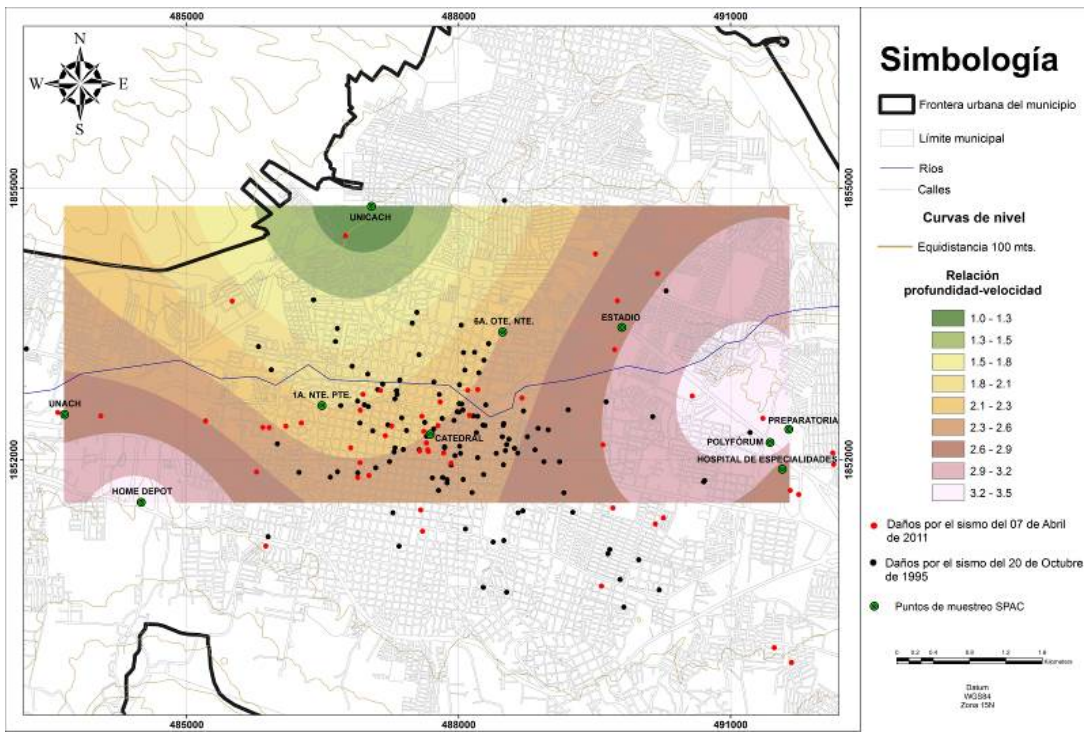

Figura 16. Relación normalizada de la velocidad de corte hasta $(720 \mathrm{~m} / \mathrm{s})$ respecto a la profundidad donde esta es alcanzada, considerando el punto más rígido (UNICACH)

En la figura 17 se muestran los daños del sismo del 7 de septiembre de 2017 colocados sobre las amplificaciones sísmicas de la microzonificación de González-Herrera et al. (2012), las cuales son coincidentes con los colores cálidos, por lo que se asume que el daño que sufre una edificación por las ondas de un sismo depende, en gran medida, del tipo de terreno sobre el cual está construida y de los materiales empleados para su construcción (Garduño et al., 2009); sin embargo, en zonas donde la vulnerabilidad de las construcciones es homogénea, el efecto de sitio es determinante (Moreno et al., 2019).

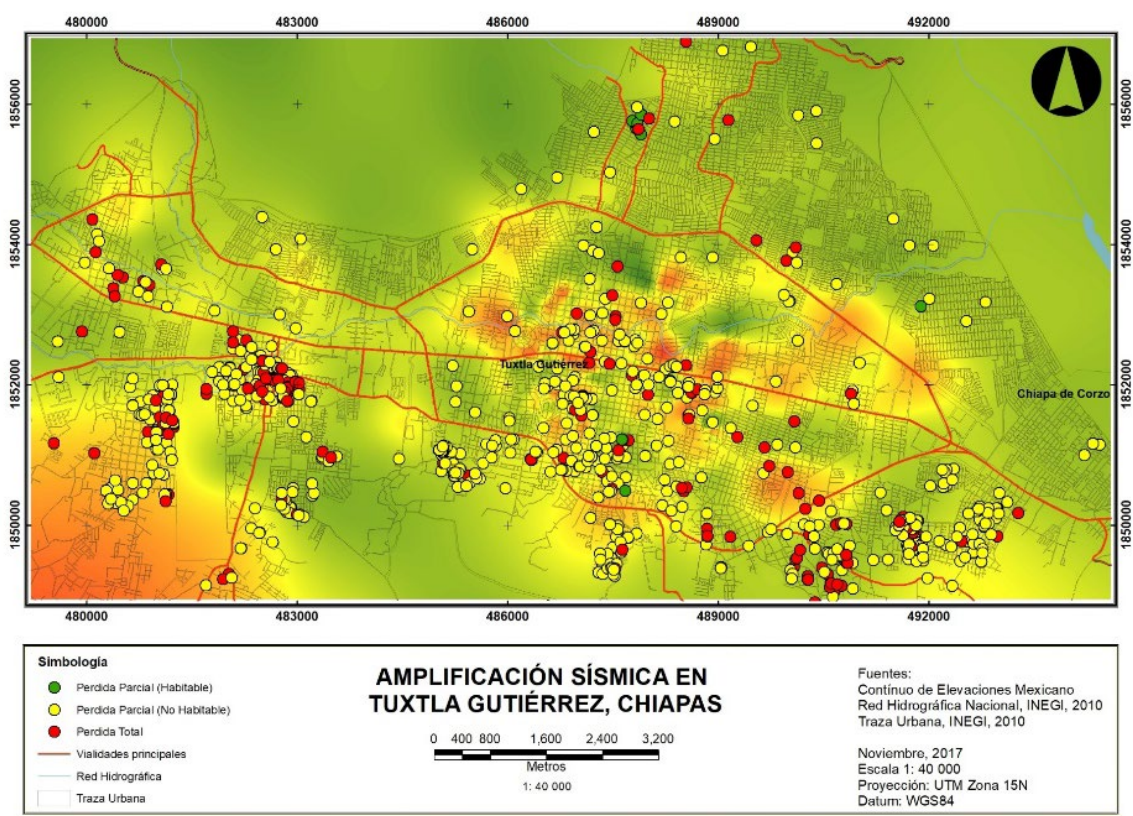

Figura 17. Localización de daños del sismo del 7 de septiembre de 2017 (puntos amarillos daños parciales y puntos rojos daños totales). Los puntos se colocan en el mapa de amplificación sísmica 


\section{Efecto de sitio empleando sismos reales en Tuxtla Gutiérrez}

Un elemento clave para valorar la importancia de la microzonificación sísmica, es determinar el efecto de sitio y los niveles de amplificación en cada parte de la ciudad. Toda microzonificación puede ser calibrada a través de distintas variables como son los sismos que se registren en la ciudad de estudio y que son registrados en las estaciones fijas o en redes temporales. Para ejemplificar esta circunstancia se desarrolló el análisis del sismo sucedido el 7 abril del 2011, evento registrado por dos estaciones acelerográficas a nivel de superficie en Tuxtla Gutiérrez; y el sismo del 7 de septiembre de 2017, registrado en cuatro puntos de la ciudad, mediante estaciones acelerográficas en superficie y otra ubicada en el primer nivel de una estructura escolar de dos niveles (tabla 1 y figura 2 ).

Para el evento del 7 de abril de 2011 la aceleración máxima presentada en la estación de la UNICACH fue de $48 \mathrm{~cm} / \mathrm{s}^{2}$ en dirección N-S y para la estación UNACH, en la misma dirección N-S, fue de $115 \mathrm{~cm} / \mathrm{s}^{2}$. Al realizar un comparativo entre las aceleraciones registradas en las dos estaciones fijas, se observa que para la estación de la UNACH la aceleración es aproximadamente 2.4 veces con respecto a la de la estación de la UNICACH para esa componente, lo cual muestra un efecto de sitio.

En la figura 18 se muestra la gráfica del cociente espectral $\mathrm{H} / \mathrm{V}$ para ruido ambiental y para el sismo del 7 de abril de 2011, se observa que la señal de la UNICACH presenta un pico predominante en la frecuencia de $8.5 \mathrm{~Hz}$ con una amplitud relativa de 4. Para la relación $\mathrm{H} / \mathrm{V}$ de la UNACH el pico predominante se observa en la frecuencia de $3.5 \mathrm{~Hz}$ con una amplificación relativa de 7 . Los cocientes espectrales $\mathrm{H} / \mathrm{V}$ del sismo, con respecto a los cocientes espectrales de los microtremores, no varían considerablemente en la forma, pero las amplitudes son mayores.

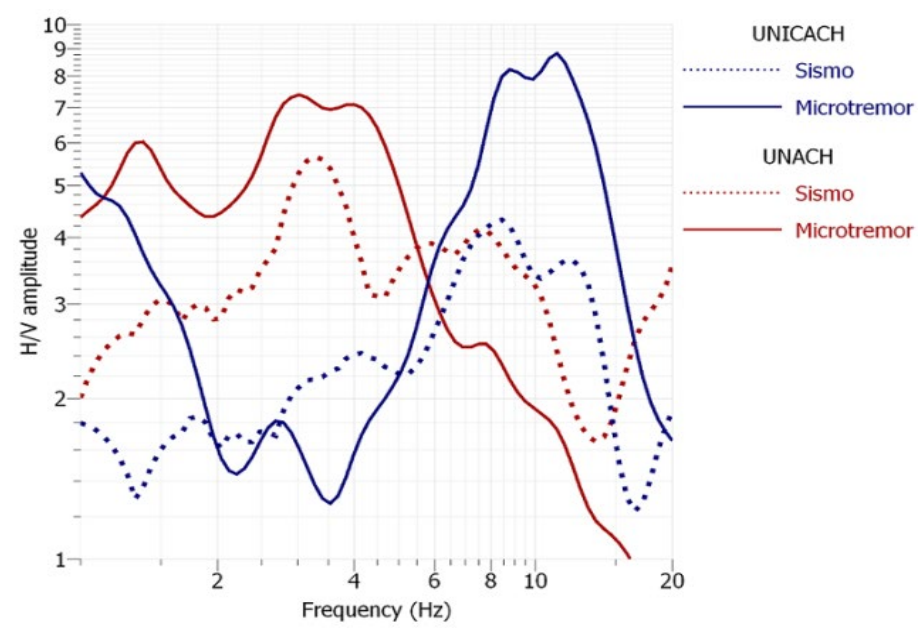

Figura 18. Gráfica amplitud de H/V de microtremores en línea continua y sismo en línea descontinúa

(Piña, 2012)

Para el evento del 7 de septiembre de 2017, al realizar un comparativo entre estas aceleraciones, se observa que para la estación N-S de la UNACH la aceleración es aproximadamente 2.9 veces con respecto a la UNICACH para la misma dirección, lo cual es similar al efecto que nos mostró el evento de abril de 2011 (2.4), no obstante, las diferencias de entre las características de estos dos eventos sísmicos. 


\section{CONCLUSIONES}

Dentro de los resultados de este trabajo se identificó el efecto de sitio como una causal determinante de daños históricos en la ciudad, lo cual se demostró tras analizar los daños de los sismos del 23 de septiembre de 1902, 21 de octubre de 1995, 7 de abril de 2011 y 7 de septiembre de 2017. Los resultados mostraron que en la ciudad se presentó un efecto de sitio con amplificaciones de la aceleración sísmica de hasta tres veces en los sitios donde se ubican estaciones acelerométricas fijas. No obstante, la predominancia de los efectos de sitio en los daños por sismo, en este trabajo se analizó la influencia de otras variables, tales como una serie de embovedados que conducen los remanentes de escurrimientos superficiales de antiguos cauces que irrigaban el río Sabinal y que se encuentran desplantados bajo la zona de estudio; así como la calidad constructiva y antigüedad de las edificaciones que fueron dañadas.

Tras analizar los daños por sismo en las construcciones de Tuxtla Gutiérrez, desde sismos históricos hasta el más reciente en 2017, se puede decir que en la ciudad existen las condiciones físicas y materiales para sufrir afectaciones y daños por sismo. Todos los sistemas constructivos observados predominantes en la ciudad (mampostería en sus diferentes variantes, y estructuras de concreto) presentaron diferentes niveles de daño, los cuales son más evidentes en diferentes regiones de la ciudad para cada sistema, siendo el centro de la ciudad y los tributarios del río Sabinal, las regiones donde hay mayor propensión de daños en las construcciones de mampostería; y el centro de la ciudad y la ladera sur para las estructuras de concreto.

Finalmente, si bien las características del sismo del 7 de septiembre de 2017 lo ubican como un evento icónico para la historia sísmica de México, en la ciudad de Tuxtla Gutiérrez, pese a los estudios técnicos históricos que fueron referidos en esta investigación y que hacían evidente el riesgo sísmico en la región, hubo una prevalencia de las patologías constructivas, debido -entre otros factores- a la falta de empleo de los estudios técnicos previos para la reducción de los vacíos del reglamento de construcción donde se enfatizaba el efecto de sitio.

\section{REFERENCIAS}

Aguilar, R (2006), "Determinación de características dinámicas en estructuras críticas de Tuxtla Gutiérrez, Chiapas". Tesis de licenciatura. Universidad Autónoma de Chiapas, Facultad de Ingeniería, Tuxtla Gutiérrez, Chiapas.

Aguilar, H R, L E Pérez-Rocha y A Jaramillo (2009), "Respuesta sísmica del terreno para Hospital bicentenario". Informe técnico Ingenieros Especialistas en Cimentaciones, S.C., México, D.F., 38 pp.

Aguilar, H R, L E Pérez-Rocha y A Jaramillo (2006), "Respuesta sísmica del terreno para Home Depot". Informe Sísmica de Suelos, S.A. de C.V., México, 46 pp.

Aguirre, J, M Rodríguez y R Vázquez (2006), "Sísmica de dispersión de ondas superficiales usando ruido sísmico. Siete mediciones en el Estado de Colima, México". Congreso Nacional de Mecánica de Suelos, Tuxtla Gutiérrez, Chiapas, 22-25 noviembre, pp. 495-504.

Aki, K (1957), "Space and time spectra of stationary stochastic waves with special reference to microtremors". Bulletin Earthquake Research Institute, Tokyo University, 35, pp. 415-457.

Aki, K y P G Richards (1980), Quantitative Seismology, W. H. Freeman, San Francisco, California. 
Alonso, G, R Cruz, F Cruz, M Ramírez, M Ruiz y J Iglesias (1999), "Resultados de la zonificación sísmica de la ciudad de Tuxtla Gutiérrez", XII Congreso Nacional de Ingeniería Sísmica, México.

Alonso, G, R Cruz, F Cruz, M Ramírez, M Ruiz y J Iglesias (1995), “Zonificación sísmica de la ciudad de Tuxtla Gutiérrez”. Informe técnico, Universidad Autónoma de Chiapas y Universidad Metropolitana, México. https://core.ac.uk/download/pdf/48394604.pdf

Arguello-Mendez, T, B Arguelles-Leon y R M Badillo-Gonzalez (2012), "Caracteristicas fisicas de la vivienda popular en la periferia urbana de Tuxtla Gutierrez, Chiapas, México", Quehacer Científico en Chiapas 1 (14) (2012) 4-13. https://dgip.unach.mx/images/pdf-revistaquehacercientifico/quehacer-cientifico-2012-jul-dic/caracteristicas fisicas vivienda popular.pdf

Atakan, K, B Brandsdóttir, P Halldórsson y G O Fridleifsson (1997), "Site Response as a Function of NearSurface Geology in the South Iceland Seismic Zone". Natural Hazards, 15(2-3), 139-164. DOI: $\underline{\text { 10.1023/A:1007902419241 }}$

Bard, P Y (1999), “Microtremor measurements: A tool for site effect estimation?", The Effects of Surface Geology on Seismic Motion, Irikura, Kudo, Okada \& Sasatani editors, Balkema, 3-1, pp. 1251-1279. https://www.researchgate.net/publication/235623097_Microtremor_measurements_A tool_for_site effect estimation

Castro, A (2001), “Cronología histórica sobre los desastres naturales y sociales en Tuxtla Gutiérrez". Páginas históricas de Chiapas, 48, México, pp. 1-2.

Chávez-García, F J y G A Montalva (2014), "Efectos de sitio para Ingenieros Geotécnicos, estudio del valle Parkway". Obras y proyectos, (16), 6-30. DOI: 10.4067/S0718-28132014000200001

Chávez-García, F J, M Rodríguez y W R Stephenson (2006), "Subsoil Structure Using SPAC Measurements along a Line". Bulletin of the Seismological Society of America, 96, 729-736. DOI: $10.1785 / 0120050141$

Chávez-García, F J, M Rodríguez y W R Stephenson (2005), “An alternative approach to the SPAC analysis of microtremors: exploiting the stationary of noise". Bulletin of the Seismological Society of America, 95-1, 277-293. DOI: 10.1785/0120030179

CENAPRED (2015), Atlas Nacional de Riesgos, CENAPRED, México, D.F. http://www.atlasnacionalderiesgos.gob.mx/index.php?option=com content\&view=article\&id=48\&I temid $=170$.

Comisión Federal de Electricidad (CFE) (2015), Manual de obras civiles diseño por sismo, Instituto de Investigaciones Eléctricas, CFE, Cuernavaca, Morelos, México.

Comisión Federal de Electricidad (CFE) (2008), Manual de obras civiles diseño por sismo, Instituto de Investigaciones Eléctricas, CFE, Cuernavaca, Morelos, México.

Ewing, M, W Jardetzky y F Press (1957), Elastic waves in layered media, Mc-Graw Hill Book Co., New York.

Figueroa, J (1986), Isosistas de grandes temblores ocurridos en la República Mexicana, Series del Instituto de Ingeniería de la UNAM, 57 pp. México. 
Figueroa, J (1973), "Sismicidad en Chiapas". Reporte técnico del Instituto de Ingeniería de la UNAM, México.

Flores, H (2004), "Método SPAC: Una alternativa para la estimación de modelos de velocidades en el Valle de México. México". Tesis de Maestría, Instituto de Geofísica, Universidad Nacional Autónoma de México, 122 pp.

Gallo, L, S Arango y A Cruz (2017), "Evaluación de los Efectos de Sitio con la Técnica de Cocientes Espectrales a partir de Vibraciones Ambientales". VIII Congreso Nacional de Ingeniería Sísmica, Barranquilla, Colombia.

García, V y G Suárez (1996), Los sismos en la historia de México, Edición Científica Universitaria, Fondo de Cultura Económica, UNAM, CIESAS, México, 718 pp.

Garduño, V H, J Chávez, J Aguirre, R Vázquez, H Mijares, I Israde, V M Hernández, M A Rodríguez y R Pérez (2009), "Zonificación de los periodos naturales de oscilación superficial en la ciudad de Pátzcuaro, Michoacán, México, con base en microtremores y estudios de paleosismología". Revista Mexicana de Ciencias Geológicas, 26-3, 623-637, México. http://www.scielo.org.mx/scielo.php?script=sci_arttext\&pid=S1026-87742009000300007

González-Herrera, R (2014), Estimación de las pérdidas económicas en las estructuras asociadas a peligro sísmico en Tuxtla Gutiérrez, Chiapas, Págs. 267. ISBN 978-607-02-5366-9. Universidad Nacional Autónoma de México, Colección Ciencia Nueva, México, https://repositorio.unam.mx/contenidos/69908.

González-Herrera, R, J C Mora, J Aguirre y D A Novelo (2013), "La estructura de velocidades y su relación con el peligro sísmico en Tuxtla Gutiérrez, Chiapas". Revista Mexicana de Ciencias Geológicas, UNAM, 30-1, ISSN 2007-2902. Juriquilla, Querétaro, México. http://www.scielo.org.mx/scielo.php?script=sci_arttext\&pid=S1026-87742013000100008

González-Herrera, R, J C Mora y J Aguirre (2012), Microzonificación sísmica de Tuxtla Gutiérrez, Chiapas, Instituto de Protección Civil para el manejo de riesgos de Desastres del estado de Chiapas, 165 p. Tuxtla Gutiérrez, Chiapas, México. https://proteccioncivil.tuxtla.gob.mx/Microzonificacion-sismica--tuxtla-gutierrez-chiapas

González-Herrera, R, J A Aguilar, J C Mora, R Palacios y J A Figueroa (2011a), “Análisis estadístico del peligro e inferencia del riesgo sísmico en el norte del estado de Chiapas". Ingeniería, Revista Académica de la FI-UADY, 16-1, pp. 51-57, México. http://www.revista.ingenieria.uady.mx/volumen16/analisis.pdf

González-Herrera, R, A Tena, J C Mora, M A Borraz, J A Aguilar, R Cruz, J A Figueroa, J A Chan, M S Ramírez, R Vera y C Narcía (2011b), El sismo de Villaflores, Chiapas, su realidad y consecuencias, Universidad de Ciencias y Artes de Chiapas, Colección Jaguar, 400 pp. Tuxtla Gutiérrez, Chiapas, México.

https://www.espacioimasd.unach.mx/libro/num15/El sismo de Villaflores Chiapas sus realidade s_y consecuencias.php 
González-Herrera, R, M A Borraz, P A Sánchez, Y De la Cruz, J A Aguilar y A Ruiz (2010), "Construcción histórica de la vulnerabilidad sísmica en Tuxtla Gutiérrez, Chiapas". XVII Congreso Nacional de Ingeniería Estructural, León, Guanajuato, 3 al 6 de noviembre, México.

Gutiérrez, C, S Miller, C Montoya y R Tapia (1991), Diagnóstico de peligro sísmico para la República Mexicana y evaluación de intensidades para sismos históricos. CENAPRED, México.

Haskell, N A (1953), “The dispersion of surface waves on multilayered media”, Bulletin of Seismological Society of America, 43, 17-34.

Haskell, N A (1960), "Crustal reflection of SH waves", Journal of Geophysical Research, 65, 4147-4150. DOI: $10.1029 / \mathrm{JZ} 065 \mathrm{i} 012 \mathrm{p} 04147$

Haskell, N A (1962), "Crustal reflection of plane P and SV waves", Journal of Geophysical Research, 67, 4751-4767. DOI: 10.1029/JZ067i012p04751

Lermo, J y F J Chávez-García (1994), “Are microtremors useful in site response evaluation?”, Bulletin of the Seismological Society of America, 83, pp. 1350-1364.

Lermo, J y F J Chávez-García (1993), "Site effect evaluation using spectral ratios with only one station", Bulletin of the Seismological Society of America, 83(5), 1574-1594.

Marsal, R J y M Mazari (1959), El subsuelo de la Ciudad de México, UNAM en segunda edición 1969.

Moreno, R, R González-Herrera, J A Paz, J A Aguilar y C U del Carpio (2019), "Effects of Sediment Thickness upon Seismic Amplification in the Urban Area of Chiapa de Corzo, Chiapas, Mexico", Earth Sciences Research Journal, Vol 23, No. 2, pp. 1-7. DOI: 10.15446/esrj.v23n2.72623

Narcía, C, J Aguilar, M Ramírez, R Cruz y R González-Herrera (2006), “El periodo natural de vibración del suelo en la ciudad de Tuxtla, Gutiérrez, Chiapas". Quehacer cientifico en Chiapas. 1-1, pp. 22-38, UNACH. Tuxtla Gutiérrez, Chiapas, México. https://www.dgip.unach.mx/images/pdf-revistaquehacercientifico/2006-ene-junio/el_periodo_natural_de_vibracion_del_.pdf

Nakamura, Y (1989), "A method for dynamic characteristics estimation of subsurface using microtremor on the ground surface". QR of RTRI 30-1, February, pp. 25-33. https://trid.trb.org/view/294184

Newmark, N y E Rosenblueth (1976), Fundamentos de Ingeniería Sísmica, $1^{\text {a }}$ edición Diana, México.

Okuwaki, R y Y Yagi (2017), "Rupture process during the Mw 8.12017 Chiapas, Mexico earthquake: shallow intraplate normal faulting by slab bending”. Geophysical Research Letters, 44, 11, 816-11,823. DOI: $\underline{10.1002 / 2017 \text { GL075956 }}$

Ordóñez, J. (2008), “Zonificación geotécnica de la ciudad de Tuxtla Gutiérrez, Chiapas”. Informe técnico, Universidad Autónoma de Chiapas, México.

Ordóñez-Ruiz, J, C Auvinet-Guichard y M Juárez-Camarena (2015), "Caracterización del subsuelo y análisis de riesgos geotécnicos asociados a las arcillas expansivas de la ciudad de Tuxtla Gutiérrez". Ingeniería Investigación y Tecnología, vol. XVI (3), julio-septiembre 453-470. http://www.scielo.org.mx/pdf/iit/v16n3/v16n3a12.pdf 
Paz Tenorio, J A, R González-Herrera, M Gómez Ramírez y J A Velasco Herrera (2017), "Metodología para elaborar mapas de susceptibilidad a procesos de remoción en masa, análisis del caso ladera sur de Tuxtla Gutiérrez, Chiapas". Revista de Investigaciones Geográficas, Año 48, No. 92, pp. 1-16. DOI: $10.14350 /$ rig.52822

Piña, J (2012), "Distribución espacial de periodos dominantes en la ciudad de Tuxtla Gutiérrez, Chiapas", Tesis de licenciatura, Facultad de Ingeniería, Universidad Nacional Autónoma de México, 107 pp. México. http://www.ptolomeo.unam.mx:8080/xmlui/handle/132.248.52.100/2668

Sánchez-Trujillo, V M, R González-Herrera, G Castañeda-Nolasco, C M García-Lara y J A Aguilar Carboney (2019), "Characterization of pathologies in housing structures. A case study in the city of Tuxtla Gutierrez, Chiapas, Mexico" Journal of Building Engineering, Vol 22, pp. 539-548. DOI: $\underline{10.1016 / j . j o b e .2019 .01 .014}$

Servicio Sismológico Nacional (SSN) (2017), "Reporte especial del sismo del 7 de septiembre de 2017”, consultado en línea el 03/04/2018, http://www.ssn.unam.mx/sismicidad/reportesespeciales/2017/SSNMX_rep_esp_20170907_Tehuantepec_M82.pdf

Sociedad Mexicana de Ingeniería Sísmica (SMIS) (2017), "Boletín Sismos Recientes". www.smis.org.mx

Thomson, W T (1950), "Transmission of elastic waves through a stratified solid medium", Journal of Applied Physics, 21, 89-93, 1950.

Tupak, E O (2009), Microzonificación sísmica, Universidad Internacional de Andalucía. UNÍA Huelva, España.

Ye, L, T Lay, Y Bai, K F Cheung y H Kanamori (2017), “The 2017 Mw 8.2 Chiapas, Mexico, earthquake: Energetic slab detachment", Geophysical Research Letters, 44, 11,824-11,832. DOI: $\underline{10.1002 / 2017 \mathrm{GL} 076085}$

Wilmer, J y L Carrillo (2009), "Estimación de los periodos naturales de vibración de viviendas de baja altura con muros de concreto", Ciencia e Ingeniería Neogranadina, Vol. 19-1, pp. 39-54. Bogotá, junio. DOI: $10.18359 / \mathrm{rcin} .309$ 